\title{
Bounds for nonlinear composite conductors via the translation method
}

\author{
B.E. Peigney ${ }^{\mathrm{a}}$, M. Peigney ${ }^{\mathrm{b}, *}$ \\ ${ }^{a}$ CEA/DIF, BP 12, 91680 Bruyères le Châtel, France \\ ${ }^{b}$ Université Paris-Est, Laboratoire Navier (UMR 8205), CNRS, Ecole des Ponts ParisTech, IFSTTAR, \\ 77455 Marne la vallée, France
}

\begin{abstract}
Hashin-Shtrikman type bounds are proposed for nonlinear isotropic composite conductors in two dimensions. Those bounds are obtained by combining the translation method with the idea of embedding the original two-dimensional problem in an extended problem of dimension 6. Invariance properties allow the evaluation of the bounds to be dramatically simplified. Explicit results are obtained for the problem of dielectric breakdown. Numerical results are given for two-phase composites governed by power-law energy functions. The obtained bounds are shown to improve on the linear comparison bounds of the HashinShtrikman type that are delivered by the Talbot-Willis (1985) approach and the Ponte Castañeda (1991) variational method.
\end{abstract}

Keywords: composite materials, nonlinear homogenization, conductivity, bounds, translation method

\section{Introduction}

This paper is concerned with bounding the effective energy of a nonlinear composite conductor in terms of the properties of its constitutive phases. A lot of work has been devoted to that topic, especially for linear composites (see e.g. the monograph by Milton (2002)). Wiener (1912) proposed general bounds on the effective conductivity that hold for any microstructure. For statically isotropic microstructures, tighter bounds have been obtained by Hashin and Shtrikman (1962) using a variational approach that employs a homogeneous 'linear comparison' medium. The Hashin-Shtrikman bounds are known to be optimal, i.e. they are achieved by certain isotropic microstructures. The variational approach of Hashin and Shtrikman (1962) has later been extended by Willis (1977) and further generalized to

\footnotetext{
${ }^{*}$ Corresponding author.

Email address: michael.peigney@polytechnique.org (M. Peigney)
} 
nonlinear composites by Talbot and Willis (1985). A related approach for bounding the effective energy of nonlinear composites has been advanced by Ponte Castañeda (1991). The method of Ponte Castañeda (1991) makes use of a linear comparison composite with the same microstructure as the given nonlinear composite. Most crucially, any bound on the effective energy of the linear comparison composite can be used to generate a corresponding bound on the energy of the nonlinear composite. In particular, nonlinear bounds that take 3-point statistics into account can be obtained (Ponte Castañeda, 1992). When used in conjunction with the linear Hashin-Shtrikman bounds, the approach of Ponte Castañeda (1991, 1992) recovers ${ }^{1}$ the bounds of Talbot and Willis (1985). Those bounds will be referred to as the linear comparison bounds in the following. In contrast with the linear case, the linear comparison bounds are generally not optimal and can a priori be improved. For viscoplastic composites, Ponte Castañeda (2012) has shown that improved bounds could be obtained via incremental homogenization. Strictly speaking, the bounds of Ponte Castañeda (2012) hold only for a special class of isotropic microstructures that are compatible with the incremental homogenization procedure (see Ponte Castañeda (2012) for details). Aside from rigorous bounds, the issue of finding estimates of the effective behavior has also received a lot of attention (Maxwell-Garnett, 1904; Bruggeman, 1935; Norris et al., 1985; Ponte Castañeda and Kailasam, 1997; Ponte Castañeda, 2002; Idiart and Ponte Castañeda, 2013). As pointed out notably by Idiart and Ponte Castañeda (2013), bounds and estimates are complementary. In particular, bounds allow for the validity of estimates to be gauged.

In this paper, we use the 'translation' method (Milton, 1990) to derive rigorous bounds that hold for the whole class of isotropic two-dimensional composites and improve on the linear comparison bounds. Originally introduced by Lurie and Cherkaev (1984) and independently by Tartar (1985); Murat and Tartar (1985), the translation method has proved to be a powerful tool in various nonlinear homogenization problems, such as bounding the stress-strain curve of composites (Milton and Serkov, 2000; Talbot and Willis, 2004; Peigney, 2005) or bounding the recoverable strains in shape memory alloys (Bhattacharya and Kohn, 1997, Peigney, 2008, 2016). For linear composites, the Hashin-Shtrikman bounds can be elegantly recovered from the translation method by embedding the original problem in a problem of higher dimension (namely, $d^{2}$ where $d$ is the dimension of the physical space). That embedding is achieved by considering simultaneously $d$ loading orientations. For nonlinear composites, combining the translation method with the idea of embedding has proved to be a successful approach notably for bounding the breakdown limit of dielectric composites or the yield limit of ideally-plastic polycrystals (Kohn and Little, 1998, Nesi et al., 1999, 2000; Garroni and Kohn, 2003). Nesi et al. (1999) proved that the linear comparison bound cannot be better than the bounds obtained from the

\footnotetext{
${ }^{1}$ except in the unusual case where the constitutive phases are governed by non strongly convex energyfunctions (Ponte Castañeda and Willis, 1993).
} 
translation method. Those authors compared the two bounds on some examples of twodimensional composites and observed that they actually coincide. In those comparisons, Nesi et al. (1999) used the translation bound obtained by embedding the original problem in $\mathbb{R}^{4}$, as considered originally by Lurie and Cherkaev (1984) and Tartar (1985); Murat and $\operatorname{Tartar}(1985)$ in the linear setting. In this paper, we consider an embedding of the original two-dimensional problem in $\mathbb{R}^{6}$, instead of $\mathbb{R}^{4}$. This is achieved by considering 3 loading orientations, instead of 2 . The corresponding bounds are expected to be stronger than the earlier translation bounds (using 2 loading orientations) because they actually apply to a smaller class of composites (namely the class of composites having the same response along 3 loading orientations, rather than 2). Although the calculations become more difficult, the bounds obtained are shown to often improve on the linear comparison bounds.

The general methodology is introduced in Sect. 2, with special emphasis on the problem of deriving lower bounds on the effective energy. The relevance of the proposed approach is demonstrated in Sect. 3 by considering some examples for which explicit results can be obtained. Embedding in $\mathbb{R}^{6}$ has the critical downside of dramatically increasing the complexity of the calculations. In Sect. 4 we use invariance properties to show that major simplifications occur when the individual phases are isotropic. The developments of Sect. 4 are essential to make the approach tractable and to be able to evaluate the bounds in practice. The proposed approach is applied in Sect. 5 to power law composites. In Sect. 6, the methodology is adapted to bound the effective energy from above.

\section{A general lower bound on the effective energy}

Consider a two-dimensional inhomogeneous electric conductor occupying a domain $\Omega$ of unit volume. The electric field $\boldsymbol{e}$ and the current density $\boldsymbol{j}$ are related by the local constitutive law

$$
\boldsymbol{j}=\frac{\partial w}{\partial \boldsymbol{e}}(\boldsymbol{e}, \boldsymbol{x})
$$

where $w$ is the convex energy-density function, depending both on $\boldsymbol{e}$ and on the location $\boldsymbol{x}$. Denoting by $\overline{\boldsymbol{e}}$ (resp. $\overline{\boldsymbol{j}}$ ) the spatial average of $\boldsymbol{e}$ (resp. $\boldsymbol{j}$ ), it can be shown (Hill, 1963 ; Willis, 1986) that $\overline{\boldsymbol{e}}$ and $\overline{\boldsymbol{j}}$ are related by the effective constitutive law

$$
\overline{\boldsymbol{\jmath}}=\frac{d w_{\text {eff }}}{d \overline{\boldsymbol{e}}}(\overline{\boldsymbol{e}})
$$

where $w_{\text {eff }}$ is the effective energy function of the composite material, defined by

$$
w_{e f f}(\overline{\boldsymbol{e}})=\inf _{\boldsymbol{e} \in \mathcal{K}(\overline{\boldsymbol{e}})} \int_{\Omega} w(\boldsymbol{e}, \boldsymbol{x}) d \omega
$$

with

$$
\mathcal{K}(\overline{\boldsymbol{e}})=\left\{\boldsymbol{e}: \Omega \mapsto \mathbb{R}^{2} \mid \boldsymbol{e}=\nabla V \text { for some } V: \Omega \mapsto \mathbb{R} \text { verifying } V(\boldsymbol{x})=\overline{\boldsymbol{e}} \cdot \boldsymbol{x} \text { on } \partial \Omega\right\} .
$$


Determining $w_{\text {eff }}(\overline{\boldsymbol{e}})$ formally amounts to solve the set of local equations

$$
\operatorname{div} \boldsymbol{j}=0 \text { in } \Omega, \boldsymbol{j}=\frac{\partial w}{\partial \boldsymbol{e}}(\boldsymbol{e}, \boldsymbol{x}), \boldsymbol{e} \in \mathcal{K}(\overline{\boldsymbol{e}}) .
$$

The problem (2.1)-2.4 arises in many physical contexts, such as thermal conductivity, magnetic permeability or diffusion. Alternatively, the local constitutive law (2.1) can be rewritten as

$$
\boldsymbol{e}=\frac{\partial w^{*}}{\partial \boldsymbol{j}}(\boldsymbol{j}, \boldsymbol{x})
$$

where the complementary energy-density function $w^{*}$ is the Legendre dual of $w$. Similarly, the effective constitutive law 2.2 can be expressed as

$$
\overline{\boldsymbol{e}}=\frac{d w_{\text {eff }}^{*}}{d \overline{\boldsymbol{\jmath}}}(\overline{\boldsymbol{\jmath}})
$$

where the effective complementary energy function $w_{\text {eff }}^{*}$ is the Legendre dual of $w_{\text {eff }}$ and satisfies

$$
w_{e f f}^{*}(\overline{\boldsymbol{\jmath}})=\inf _{\boldsymbol{j} \in \mathcal{S}(\overline{\boldsymbol{\jmath}})} \int_{\Omega} w^{*}(\boldsymbol{j}, \boldsymbol{x}) d \omega
$$

with

$$
\mathcal{S}(\overline{\boldsymbol{\jmath}})=\left\{\boldsymbol{j}: \Omega \mapsto \mathbb{R}^{2} \mid \operatorname{div} \boldsymbol{j}=0 \text { in } \Omega ; \boldsymbol{j}(\boldsymbol{x}) \cdot \boldsymbol{n}=\overline{\boldsymbol{\jmath}} \cdot \boldsymbol{n} \text { on } \partial \Omega\right\} .
$$

This paper is concerned with deriving bounds on $w_{\text {eff }}$ and $w_{\text {eff }}^{*}$, with special emphasis on the case where the overall behavior is isotropic. Since $w_{\text {eff }}$ and $w_{\text {eff }}^{*}$ are Legendre duals of each other, a lower (resp. upper) bound on $w_{\text {eff }}$ translates as an upper (resp. lower) bound on $w_{e f f}^{*}$, and vice versa. In order to simplify the presentation, we mainly focus on lower bounds on $w_{\text {eff }}$. The issue of deriving upper bounds on $w_{\text {eff }}$ is addressed in Sect. 6 .

\subsection{Extended effective energy}

Consider $n$ distinct values $\overline{\boldsymbol{e}}_{1}, \cdots, \overline{\boldsymbol{e}}_{n}$ and let $\boldsymbol{e}_{i}(\boldsymbol{x})$ be the solution of (2.4) for $\overline{\boldsymbol{e}}=\overline{\boldsymbol{e}}_{i}$. It can be seen from (2.3) that the extended field $\boldsymbol{E}=\left(\boldsymbol{e}_{1}, \cdots, \boldsymbol{e}_{n}\right)$ solves the minimization problem

$$
\inf _{\boldsymbol{E} \in \mathcal{K}(\overline{\boldsymbol{E}})} \int_{\Omega} \sum_{i=1}^{n} w\left(\boldsymbol{e}_{i}, \boldsymbol{x}\right) d \omega
$$

where $\overline{\boldsymbol{E}}=\left(\overline{\boldsymbol{e}}_{1}, \cdots, \overline{\boldsymbol{e}}_{n}\right)$ and

$$
\mathcal{K}(\overline{\boldsymbol{E}})=\left\{\left(\boldsymbol{e}_{1}, \cdots, \boldsymbol{e}_{n}\right): \boldsymbol{e}_{i}=\nabla V_{i} \text { for some } V_{i}: \Omega \mapsto \mathbb{R} \text { verifying } V_{i}(\boldsymbol{x})=\overline{\boldsymbol{e}}_{i} \cdot \boldsymbol{x} \text { on } \partial \Omega\right\} .
$$

In view of 2.6, it is convenient to introduce the extended energy $W$ defined as

$$
W(\boldsymbol{E}, \boldsymbol{x})=\sum_{i=1}^{n} w\left(\boldsymbol{e}_{i}, \boldsymbol{x}\right) .
$$


Similarly, we define the extended effective energy $W_{\text {eff }}$ by

$$
W_{\text {eff }}(\overline{\boldsymbol{E}})=\sum_{i=1}^{n} w_{\text {eff }}\left(\overline{\boldsymbol{e}}_{i}\right) .
$$

Combining (2.3) and 2.6) leads to

$$
W_{\text {eff }}(\overline{\boldsymbol{E}})=\inf _{\boldsymbol{E} \in \mathcal{K}(\overline{\boldsymbol{E}})} \int_{\Omega} W(\boldsymbol{E}, \boldsymbol{x}) d \omega .
$$

The variational structure 2.8 is similar to that of $w_{\text {eff }}$ in 2.3 , except it is defined in terms of higher dimensional fields.

\subsection{Translated bounds on the extended energy}

We now use the translation method to generate bounds on $W_{\text {eff. }}$. Let $U(\boldsymbol{E})$ be a given function (referred to in the following as a comparison potential) and consider the Legendre transform

$$
(W-U)^{*}(\boldsymbol{T}, \boldsymbol{x})=\sup _{\boldsymbol{E}} \boldsymbol{E} \cdot \boldsymbol{T}-W(\boldsymbol{E}, \boldsymbol{x})+U(\boldsymbol{E})
$$

for any $\boldsymbol{T}$ in $\mathbb{R}^{2 n}$. The dot $\cdot$ in 2.9 denotes the Euclidean scalar product in $\mathbb{R}^{2 n}$. It follows from 2.9 that

$$
W(\boldsymbol{E}, \boldsymbol{x}) \geq \boldsymbol{E} \cdot \boldsymbol{T}+U(\boldsymbol{E})-(W-U)^{*}(\boldsymbol{T}, \boldsymbol{x})
$$

for any $\boldsymbol{E}$ and $\boldsymbol{T}$ in $\mathbb{R}^{2 n}$. Taking $\boldsymbol{T}$ independent on $\boldsymbol{x}$ and integrating 2.10$)$ over the domain $\Omega$, we find

$$
\int_{\Omega} W(\boldsymbol{E}, \boldsymbol{x}) d \omega \geq \overline{\boldsymbol{E}} \cdot \boldsymbol{T}+\int_{\Omega} U(\boldsymbol{E}) d \omega-\int_{\Omega}(W-U)^{*}(\boldsymbol{T}, \boldsymbol{x}) d \omega
$$

for any $\boldsymbol{E} \in \mathcal{K}(\overline{\boldsymbol{E}})$. Assume now that the comparison potential $U$ is quasiconvex, i.e. satisfies

$$
\int_{\Omega} U(\boldsymbol{E}) d \omega \geq U(\overline{\boldsymbol{E}}) \text { for any } \boldsymbol{E} \in \mathcal{K}(\overline{\boldsymbol{E}}) .
$$

Replacing in 2.11) yields

$$
\int_{\Omega} W(\boldsymbol{E}, \boldsymbol{x}) d \omega \geq \overline{\boldsymbol{E}} \cdot \boldsymbol{T}+U(\overline{\boldsymbol{E}})-\int_{\Omega}(W-U)^{*}(\boldsymbol{T}, \boldsymbol{x}) d \omega
$$

for any $\boldsymbol{E} \in \mathcal{K}(\overline{\boldsymbol{E}})$. Since the right-hand side of 2.13 is independent on $\boldsymbol{E}$, taking the infimum over $\boldsymbol{E} \in \mathcal{K}(\overline{\boldsymbol{E}})$ gives

$$
W_{e f f}(\overline{\boldsymbol{E}}) \geq \overline{\boldsymbol{E}} \cdot \boldsymbol{T}+U(\overline{\boldsymbol{E}})-\int_{\Omega}(W-U)^{*}(\boldsymbol{T}, \boldsymbol{x}) d \omega .
$$

The right-hand side of (2.14) is thus a lower bound on $W_{\text {eff }}$. Each choice of comparison potential $U$ generates a corresponding bound. However, $U$ should be carefully chosen for the bound (2.14) to be meaningful. In that regard, a first requirement is that $(W-$ $U)^{*}(\boldsymbol{T}, \boldsymbol{x})<+\infty$, which sets restrictions on the behavior of $U$ at infinity. 


\subsection{The isotropic case}

The bound (2.14) applies to any microstructure. We assume from now on that the given composite is isotropic, in which case $w_{\text {eff }}$ only depends on the electric field $\overline{\boldsymbol{e}}$ through its norm $\bar{e}$. In other words, there exists a function $\phi_{\text {eff }}$ such that

$$
w_{\text {eff }}(\overline{\boldsymbol{e}})=\phi_{\text {eff }}(\bar{e}) .
$$

Take $\overline{\boldsymbol{E}}=\left(\overline{\boldsymbol{e}}_{1}, \cdots, \overline{\boldsymbol{e}}_{n}\right)$ such that $\overline{\boldsymbol{e}}_{1}, \cdots, \overline{\boldsymbol{e}}_{n}$ have the same norm (denoted by $\left.\bar{e}\right)$, i.e.

$$
\bar{e}_{1}=\cdots=\bar{e}_{n}=\bar{e} .
$$

We have

$$
w_{\text {eff }}\left(\overline{\boldsymbol{e}}_{1}\right)=\cdots=w_{\text {eff }}\left(\overline{\boldsymbol{e}}_{n}\right)=\phi_{\text {eff }}(\bar{e}),
$$

hence Eq. 2.14 implies the following lower bound on $\phi_{\text {eff }}(\bar{e})$ :

$$
\phi_{\text {eff }}(\bar{e}) \geq \frac{1}{n}\left(\overline{\boldsymbol{E}} \cdot \boldsymbol{T}+U(\overline{\boldsymbol{E}})-\int_{\Omega}(W-U)^{*}(\boldsymbol{T}, \boldsymbol{x}) d \omega\right) .
$$

In the derivation exposed, the isotropy is captured only by the relation (2.17), i.e. by stating that the effective energy $w_{\text {eff }}$ takes the same value along $n$ prescribed directions having the same norm. For linear materials, the condition (2.17) fully characterizes isotropy as soon as $n \geq 2$ (at least for well-chosen directions $\boldsymbol{e}_{i}$ ). For nonlinear behaviour, however, the relation (2.17) is a necessary but not sufficient condition for isotropy to occur. In such case, the bound delivered by (2.18) is expected to become tighter as $n$ increases.

Nesi et al. (1999) studied bounds of the form (2.18) with $n=2$. The comparison potential $U$ was chosen as the determinant in $\mathbb{R}^{2}$. The resulting translated bound was shown by Nesi et al. (1999) to often coincide with the linear comparison bound. In this paper, we take $n=3$ i.e. we consider 3 loading orientations instead of 2 . The resulting bounds are expected to be sharper than the translation bounds of Nesi et al. (1999) because they apply to a smaller class of composites, namely the class of composites having the same response along 3 different orientations rather than 2 . In more detail, we consider comparison potentials of the form

$$
U(\boldsymbol{E})=\alpha V(\boldsymbol{E})
$$

where $\alpha$ is a parameter and

$$
V(\boldsymbol{E})=\sum_{\substack{i, j=1 \\ i<j}}^{3}\left(u_{i} v_{j}-u_{j} v_{i}\right)
$$


In 2.20$),\left(u_{i}, v_{i}\right)$ denotes the components of the vector $\boldsymbol{e}_{i}$ in $\mathbb{R}^{2}$, so that

$$
\boldsymbol{E}=\left(u_{1}, v_{1}, u_{2}, v_{2}, u_{3}, v_{3}\right) .
$$

For any fixed $i$ and $j$, the determinant $u_{i} v_{j}-u_{j} v_{i}$ is known to be a null Lagrangian, i.e. it satisfies 2.12) as an equality. Consequently, the function 2.19) is also a null Lagrangian for any value of $\alpha$. The potential $U$ in 2.19$)$ is quadratic in $\boldsymbol{E}$ and can be written in the form

$$
U(\boldsymbol{E})=\frac{1}{2} \alpha \boldsymbol{E} \cdot \boldsymbol{M} \cdot \boldsymbol{E}
$$

where $\mathcal{M}$ is the symmetric matrix given by

$$
\mathcal{M}=\left(\begin{array}{rrrrrr}
0 & 0 & 0 & 1 & 0 & 1 \\
0 & 0 & -1 & 0 & -1 & 0 \\
0 & -1 & 0 & 0 & 0 & 1 \\
1 & 0 & 0 & 0 & -1 & 0 \\
0 & -1 & 0 & -1 & 0 & 0 \\
1 & 0 & 1 & 0 & 0 & 0
\end{array}\right)
$$

For later reference, we note that the eigenvalues of $\mathcal{M}$ are $\pm \sqrt{3}$ and 0 .

We will use (2.18) with

$$
\overline{\boldsymbol{E}}=\bar{e} \boldsymbol{N}
$$

where

$$
\boldsymbol{N}=\frac{1}{2}(1,-\sqrt{3},-1,-\sqrt{3},-2,0) .
$$

The vector $\overline{\boldsymbol{E}}=\bar{e} \boldsymbol{N}$ satisfies 2.16 and the three corresponding directions $\overline{\boldsymbol{e}}_{i}$ make a $\pi / 3$ angle between each other (Fig. 1). The main motivation for the choice (2.22) is that it allows the linear Hashin-Shtrikman bound to be recovered, as will be seen in Sect. 3.1. It can easily be verified that $\boldsymbol{N}$ is an eigenvector of $\mathcal{M}$ for the eigenvalue $-\sqrt{3}$. Therefore, using 2.18 with $\overline{\boldsymbol{E}}$ of the form 2.22, we obtain

$$
\phi_{\text {eff }}(\bar{e}) \geq \frac{\bar{e}}{3} \boldsymbol{N} \cdot \boldsymbol{T}-\frac{\sqrt{3}}{2} \alpha \bar{e}^{2}-\frac{1}{3} \int_{\Omega}(W-\alpha V)^{*}(\boldsymbol{T}, \boldsymbol{x}) d \omega .
$$

The bound 2.24 holds for any $\alpha \in \mathbb{R}$ and $\boldsymbol{T} \in \mathbb{R}^{6}$, hence

$$
\phi_{\text {eff }}(\bar{e}) \geq \sup _{\alpha \in \mathbb{R}, \boldsymbol{T} \in \mathbb{R}^{6}}\left\{\frac{\bar{e}}{3} \boldsymbol{N} \cdot \boldsymbol{T}-\frac{\sqrt{3}}{2} \alpha \bar{e}^{2}-\frac{1}{3} \int_{\Omega}(W-\alpha V)^{*}(\boldsymbol{T}, \boldsymbol{x}) d \omega\right\} .
$$

Calculations can be made simpler if the vector $\boldsymbol{T}$ in 2.25 is restricted to be parallel to $\boldsymbol{N}$, i.e. of the form $\boldsymbol{T}=\tau \boldsymbol{N}$. This generates the following lower bound:

$$
\phi_{\text {eff }}(\bar{e}) \geq \sup _{\alpha \in \mathbb{R}, \tau \in \mathbb{R}}\left\{\bar{e} \tau-\frac{\sqrt{3}}{2} \alpha \bar{e}^{2}-\frac{1}{3} \int_{\Omega}(W-\alpha V)^{*}(\tau \boldsymbol{N}, \boldsymbol{x}) d \omega\right\} .
$$




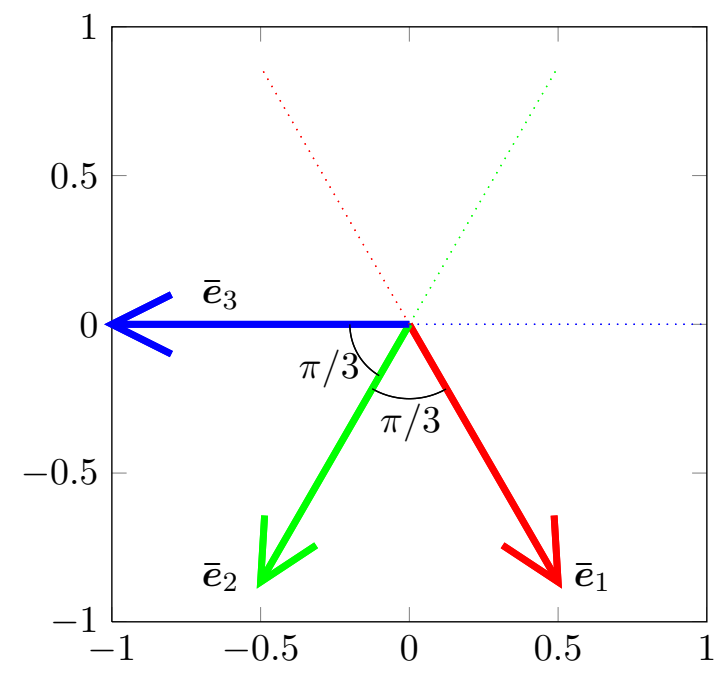

Figure 1: The three directions $\left(\overline{\boldsymbol{e}}_{1}, \overline{\boldsymbol{e}}_{2}, \overline{\boldsymbol{e}}_{3}\right)$ constituting the vector $\overline{\boldsymbol{E}}$ used in the calculation of the bounds. For an isotropic composite, the effective energies $w_{\text {eff }}\left(\overline{\boldsymbol{e}}_{1}\right), w_{\text {eff }}\left(\overline{\boldsymbol{e}}_{2}\right), w_{\text {eff }}\left(\overline{\boldsymbol{e}}_{3}\right)$ take the same value.

In general, the bound (2.26) is weaker than the bound (2.25). We will show in Sect 4 that the two bounds actually coincide if all the constitutive phases have an isotropic behavior. It should also be noted that the bounds $(2.25)$ and $(2.26)$ are meaningful only if $w(\boldsymbol{e}, \boldsymbol{x})$ has faster than quadratic growth in $\boldsymbol{e}$ (so as to ensure that $(W-\alpha V)^{*}(\tau \boldsymbol{N}, \boldsymbol{x})$ remains finite).

In the next section, the relevance of the bounds 2.25 and $(2.26)$ is demonstrated on some examples for which exact expressions can be obtained.

\section{Exact results}

\subsection{The linear case}

It is insightful to first consider the case where $w(\boldsymbol{e}, \boldsymbol{x})=\frac{1}{2} \boldsymbol{e} \cdot \boldsymbol{\sigma}(\boldsymbol{x}) \cdot \boldsymbol{e}$ and $\boldsymbol{\sigma}(\boldsymbol{x})$ is symmetric positive definite. The corresponding extended energy $W(\boldsymbol{E}, \boldsymbol{x})$ is equal to $\frac{1}{2} \boldsymbol{E}$. $\boldsymbol{\Sigma}(\boldsymbol{x}) \cdot \boldsymbol{E}$ where $\boldsymbol{\Sigma}(\boldsymbol{x})$ has the block structure

$$
\boldsymbol{\Sigma}(\boldsymbol{x})=\left(\begin{array}{lll}
\boldsymbol{\sigma}(\boldsymbol{x}) & 0 & 0 \\
0 & \boldsymbol{\sigma}(\boldsymbol{x}) & 0 \\
0 & 0 & \boldsymbol{\sigma}(\boldsymbol{x})
\end{array}\right)
$$

For an isotropic composite, the effective energy $w_{\text {eff }}(\overline{\boldsymbol{e}})$ can be written as $\frac{1}{2} \sigma_{\text {eff }} \bar{e}^{2}$ where $\sigma_{\text {eff }}$ is the effective conductivity. A lower bound on $\sigma_{\text {eff }}$ can be obtained by using the prescription 2.25) with $\bar{e}=1$. Provided that $\boldsymbol{\Sigma}(\boldsymbol{x})-\alpha \boldsymbol{M}$ is positive definite, $(W-$ 
$\alpha V)^{*}(\boldsymbol{T}, \boldsymbol{x})$ is finite and given by

$$
(W-\alpha V)^{*}(\boldsymbol{T}, \boldsymbol{x})=\frac{1}{2} \boldsymbol{T} \cdot(\boldsymbol{\Sigma}(\boldsymbol{x})-\alpha \mathcal{M})^{-1} \cdot \boldsymbol{T} .
$$

It follows from (2.24) that

$$
\sigma_{e f f} \geq \frac{2}{3} \boldsymbol{N} \cdot \boldsymbol{T}-\sqrt{3} \alpha-\frac{1}{3} \boldsymbol{T} \cdot\left[\int_{\Omega}(\boldsymbol{\Sigma}(\boldsymbol{x})-\alpha \mathcal{M})^{-1} d \omega\right] \cdot \boldsymbol{T} .
$$

Maximizing the right hand side of (3.1) with respect to $\boldsymbol{T}$, we obtain

$$
\sigma_{e f f} \geq \frac{1}{3} \boldsymbol{N} \cdot\left[\int_{\Omega}(\boldsymbol{\Sigma}(\boldsymbol{x})-\alpha \mathcal{M})^{-1} d \omega\right]^{-1} \cdot \boldsymbol{N}-\sqrt{3} \alpha .
$$

We note that the value of $\boldsymbol{T}$ realizing the maximum in (3.1) is

$$
\boldsymbol{T}=\left[\int_{\Omega}(\boldsymbol{\Sigma}(\boldsymbol{x})-\alpha \mathcal{M})^{-1} d \omega\right]^{-1} \cdot \boldsymbol{N} .
$$

Let us specialize the bound $(3.2)$ in the case where $\boldsymbol{\sigma}(\boldsymbol{x})$ is isotropic, i.e. of the form $\sigma(\boldsymbol{x}) \mathbf{1}$ where $\sigma(\boldsymbol{x})>0$ and $\mathbf{1}$ is the identity matrix in $\mathbb{R}^{2}$. Since $(\boldsymbol{\Sigma}(\boldsymbol{x})-\alpha \boldsymbol{M}) \cdot \boldsymbol{N}=$ $(\sigma(\boldsymbol{x})+\sqrt{3} \alpha) \boldsymbol{N}$, a direct calculation from $(3.2)$ leads to

$$
\sigma_{e f f} \geq\left[\int_{\Omega} \frac{1}{\sigma(\boldsymbol{x})+\sqrt{3} \alpha} d \omega\right]^{-1}-\sqrt{3} \alpha .
$$

The relation (3.4) holds provided that $\boldsymbol{\Sigma}(\boldsymbol{x})-\alpha \boldsymbol{\mathcal { M }}$ is positive definite. As noted in Sect. 2.3 . the eigenvalues of $\mathcal{M}$ are 0 and $\pm \sqrt{3}$. Hence $\boldsymbol{\Sigma}(\boldsymbol{x})-\alpha \boldsymbol{M}$ is positive definite if $|\alpha|<\sigma(\boldsymbol{x}) / \sqrt{3}$. Letting $\alpha$ tend towards $\inf \boldsymbol{x} \sigma(\boldsymbol{x}) / \sqrt{3}$ from below in (3.4), we obtain

$$
\sigma_{e f f} \geq\left[\int_{\Omega} \frac{1}{\sigma(\boldsymbol{x})+\inf _{\boldsymbol{x}} \sigma(\boldsymbol{x})} d \omega\right]^{-1}-\inf _{\boldsymbol{x}} \sigma(\boldsymbol{x}) .
$$

The formula (3.5) corresponds to the celebrated bound of Hashin and Shtrikman (1962). For instance, in the case of a two-phase composite, 3.5 specializes as

$$
\sigma_{e f f} \geq c_{1} \sigma_{1}+c_{2} \sigma_{2}-\frac{c_{1} c_{2}\left(\sigma_{1}-\sigma_{2}\right)^{2}}{\sigma_{1}+c_{2} \sigma_{1}+c_{1} \sigma_{2}}
$$

where $\sigma_{i}$ and $c_{i}$ are respectively the conductivity and the volume fraction of phase $i$. In (3.6), the phases are labelled in such fashion that $\sigma_{1}<\sigma_{2}$.

The fact that the linear Hashin-Shtrikman bound is recovered allows us to check that the choice 2.22 of $\overline{\boldsymbol{E}}$ is consistent with the potentials $U$ considered. Others choice would do, but $\overline{\boldsymbol{E}}$ cannot be taken in a completely arbitrary fashion (the important point is that $\mathcal{M} \cdot \overline{\boldsymbol{E}}=-\sqrt{3} \overline{\boldsymbol{E}})$.

In the case of linear isotropic constitutive materials, observe from (3.3) that the optimal value of $\boldsymbol{T}$ is parallel to $\boldsymbol{N}$, hence nothing is lost by considering (2.26) instead of (2.25). As will be shown in Sect. 4, that property remains true in the nonlinear case. 


\subsection{Dielectric breakdown in a two-phase composite}

Consider a two-phase nonlinear composite in which the energy $w_{i}$ of phase $i(i=1,2)$ is given by

$$
\begin{gathered}
w_{1}(\boldsymbol{e})= \begin{cases}0 & \text { if } e \leq 1, \\
+\infty & \text { if } e>1 .\end{cases} \\
w_{2}(\boldsymbol{e})= \begin{cases}0 & \text { if } e=0, \\
+\infty & \text { otherwise }\end{cases}
\end{gathered}
$$

Phase 1 behaves as a perfect dielectric so long as $e \leq 1$, and breaks down when $e$ reaches

1. Phase 2 is a perfect conductor. Assuming overall isotropy, the effective energy $w_{\text {eff }}(\overline{\boldsymbol{e}})$ takes the form

$$
w_{\text {eff }}(\overline{\boldsymbol{e}})= \begin{cases}0 & \text { if } \bar{e} \leq k_{\text {eff }} \\ +\infty & \text { if } \bar{e}>k_{\text {eff }}\end{cases}
$$

i.e. the composite material behaves as a perfect dielectric as long as $\bar{e}<k_{\text {eff }}$ and breaks down when the magnitude of the electric field $\bar{e}$ reaches the critical value $k_{\text {eff. For later }}$ reference, we note that 3.9 corresponds to an effective complementary energy $w_{\text {eff }}^{*}$ of the form

$$
w_{\text {eff }}^{*}(\bar{\jmath})=k_{\text {eff }} \bar{\jmath} .
$$

where $\bar{\jmath}$ is the magnitude of $\bar{\jmath}$. The parameter $k_{\text {eff }}$ in $(3.9)$ and 3.10$)$ is referred to as the effective strength of the composite dielectric. The formula $(2.26)$ is now use to bound $k_{\text {eff. }}$ Denoting the volume fraction of phase $i$ by $c_{i}$, Eqs. (2.26) and (3.9) imply that any $k$ in $\left[0, k_{\text {eff }}\right]$ satisfies

$$
0 \geq k \tau-\frac{\sqrt{3}}{2} \alpha k^{2}-\sum_{i=1}^{2} \frac{c_{i}}{3}\left(W_{i}-\alpha V\right)^{*}(\tau \boldsymbol{N}) \quad \forall \alpha>0, \tau \geq 0
$$

with

$$
\left(W_{i}-\alpha V\right)^{*}(\tau \boldsymbol{N})=\sup _{\boldsymbol{E}=\left(\boldsymbol{e}_{\mathbf{1}}, \boldsymbol{e}_{\mathbf{2}}, \boldsymbol{e}_{\mathbf{3}}\right)}\left\{\tau \boldsymbol{N} \cdot \boldsymbol{E}+\frac{1}{2} \alpha \boldsymbol{E} \cdot \boldsymbol{M} \cdot \boldsymbol{E}-\sum_{j=1}^{3} w_{i}\left(\boldsymbol{e}_{\boldsymbol{j}}\right)\right\} .
$$

From 3.7 and 3.8 we have

$$
\left(W_{1}-\alpha V\right)^{*}(\tau \boldsymbol{N})=\sup _{\substack{\left.\boldsymbol{E} \boldsymbol{e}_{\mathbf{1}}, \boldsymbol{e}_{\mathbf{2}}, \boldsymbol{e}_{\mathbf{3}}\right) \\ e_{i} \leq 1}}\left\{\tau \boldsymbol{N} \cdot \boldsymbol{E}+\frac{1}{2} \alpha \boldsymbol{E} \cdot \boldsymbol{M} \cdot \boldsymbol{E}\right\}
$$


and $\left(W_{2}-\alpha V\right)^{*}(\tau \boldsymbol{N})=0$. We can easily get rid of the parameter $\alpha$ in (3.11): For any non-negative $\alpha$, observe indeed from 3.13 that $\left(W_{1}-\alpha V\right)^{*}(\tau \boldsymbol{N})=\alpha g^{*}(\tau / \alpha)$ where $g^{*}$ is defined for $\tau^{\prime}>0$ by

$$
g^{*}\left(\tau^{\prime}\right)=\sup _{\substack{\boldsymbol{E}=\left(\boldsymbol{e}_{\mathbf{1}}, \boldsymbol{e}_{\mathbf{2}}, \boldsymbol{e}_{\mathbf{3}}\right) \\ e_{i} \leq 1}}\left\{\tau^{\prime} \boldsymbol{N} \cdot \boldsymbol{E}+\frac{1}{2} \boldsymbol{E} \cdot \boldsymbol{M} \cdot \boldsymbol{E}\right\}
$$

Further setting

we have

$$
P(k, \tau)=k \tau-\frac{\sqrt{3}}{2} k^{2}-\frac{c_{1}}{3} g^{*}(\tau)
$$

$$
\begin{aligned}
\sup _{\alpha>0, \tau \geq 0} k \tau-\frac{\sqrt{3}}{2} \alpha k^{2}-\sum_{i=1}^{2} \frac{c_{i}}{3}\left(W_{i}-\alpha V\right)^{*}(\tau \boldsymbol{N}) & =\sup _{\alpha>0, \tau \geq 0} \alpha P\left(k, \frac{\tau}{\alpha}\right) \\
& =\sup _{\alpha>0, \tau^{\prime} \geq 0} \alpha P\left(k, \tau^{\prime}\right)
\end{aligned}
$$

Hence the condition (3.11) is equivalent to

$$
0 \geq \sup _{\tau \geq 0} P(k, \tau) .
$$

Any $k$ verifying $0<\sup _{\tau \geq 0} P(k, \tau)$ is necessary above $k_{\text {eff. Therefore, an upper bound } \tilde{k}}$ on $k_{\text {eff }}$ is given by

$$
\tilde{k}=\inf \left\{k: 0<\sup _{\tau \geq 0} P(k, \tau)\right\} .
$$

Evaluating $\tilde{k}$ requires some details on the function $g^{*}$ introduced in 3.14 . Note that $g^{*}$ is convex because it is defined as the pointwise supremum of a family of linear functions. The constrained quadratic optimization problem (3.14) can be solved in closed form as detailed in Appendix A. Setting $\tau_{0}=1+\sqrt{3}$, it is shown in Appendix A that:

$$
g^{*}(\tau)= \begin{cases}\tau(\cos \theta-\sqrt{3} \sin \theta+1)+2(\cos \theta+1) \sin \theta & \text { if } 0 \leq \tau \leq \tau_{0}, \\ 3\left(\tau-\frac{\sqrt{3}}{2}\right) & \text { if } \tau \geq \tau_{0},\end{cases}
$$

where $\theta$ is characterized by the conditions

$$
\begin{aligned}
& \sin \theta= \begin{cases}\frac{\sqrt{3}}{2} & \text { if } \tau=0 \\
\frac{2}{\tau}\left(2 \cos ^{2} \theta-1+\cos \theta\right)-\sqrt{3} \cos \theta & \text { if } \tau>0\end{cases} \\
& \cos \theta=z-\frac{1}{2}+\frac{\tau}{2 \sqrt{3}},
\end{aligned}
$$


and $z$ is the largest root of the cubic equation

$$
8 z^{3}-6 z+\frac{\sqrt{3} \tau^{3}}{9}-2=0
$$
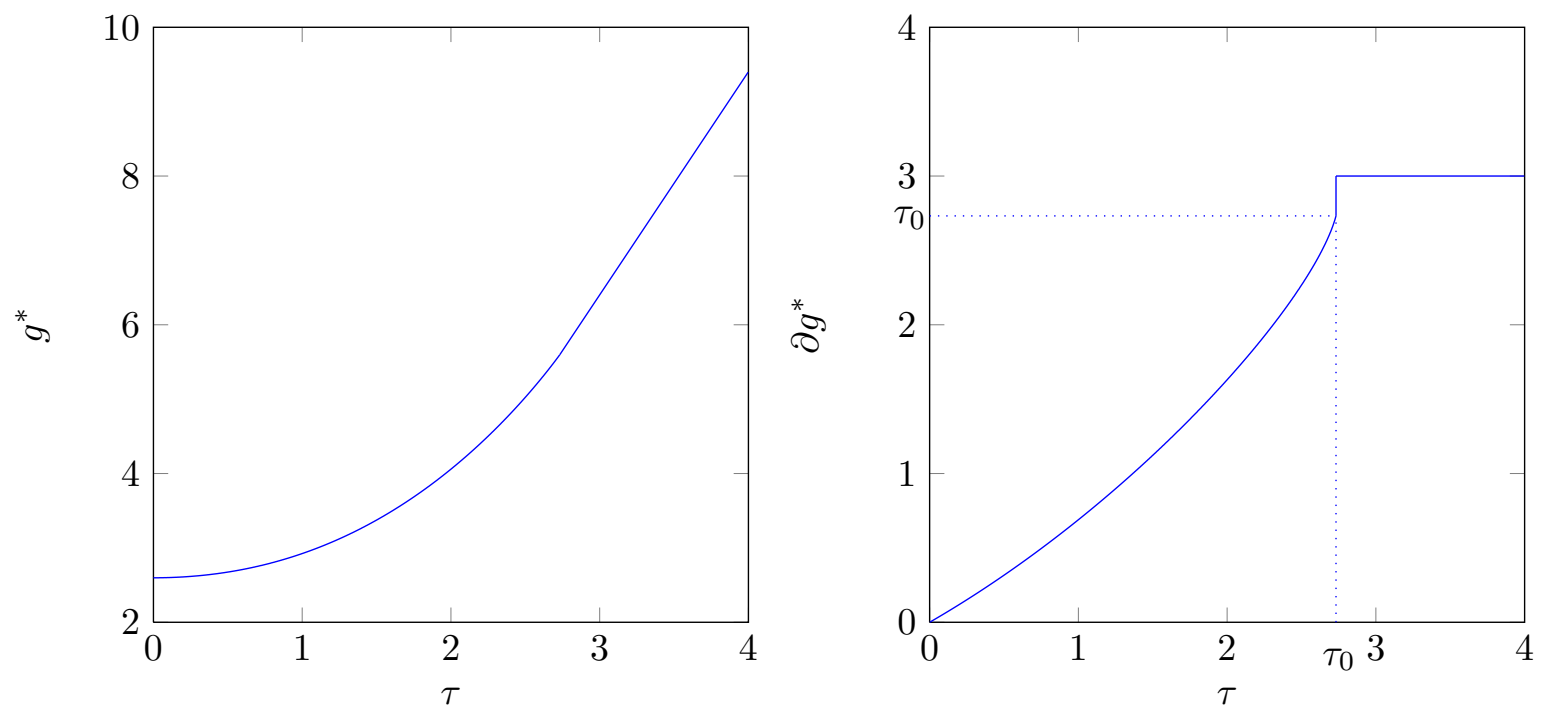

Figure 2: Plots of the function $g^{*}$ and of its subdifferential $\partial g^{*}$.

The function $g^{*}$ is depicted in Fig. 2(left). It follows from (3.16)-(3.18) that $g^{*}$ is differentiable for $\tau \neq \tau_{0}$, with a derivative given by

$$
\frac{d g^{*}}{d \tau}(\tau)= \begin{cases}\cos \theta-\sqrt{3} \sin \theta+1 & \text { for } 0 \leq \tau<\tau_{0} \\ 3 & \text { for } \tau>\tau_{0}\end{cases}
$$

At $\tau=\tau_{0}, g^{*}$ is subdifferentiable $($ Rockafellar, 1970 $)$ and we have

$$
\partial g^{*}\left(\tau_{0}\right)=\left[\lim _{\tau \longrightarrow \tau_{0}^{-}} \frac{d g^{*}}{d t}(\tau), \lim _{\tau \longrightarrow \tau_{0}^{+}} \frac{d g^{*}}{d t}(\tau)\right]=\left[\tau_{0}, 3\right] .
$$

The subdifferential $\partial g^{*}$ is represented in Fig. 2(right). The nondifferentiability of $g^{*}$ at $\tau=\tau^{0}$ corresponds to a (hardly noticeable) discontinuity in the slope of the curve $\tau \mapsto g^{*}(\tau)$ shown in Fig. 2(left).

We are now in a position to evaluate the bound $\tilde{k}$ in 3.15 . By 3.16 we have

$$
\sup _{\tau \geq \tau_{0}} P(k, \tau)= \begin{cases}+\infty & \text { if } k>c_{1} \\ P\left(k, \tau_{0}\right) & \text { if } k \leq c_{1}\end{cases}
$$


Hence 3.15 can be rewritten as

$$
\tilde{k}=\inf \left\{k \leq c_{1}: 0<\sup _{0 \leq \tau \leq \tau_{0}} P(k, \tau)\right\} .
$$

Since $P$ is continuous in $(k, \tau)$, the function $\sup _{0 \leq \tau \leq \tau_{0}} P(k, \tau)$ is continuous in $k$. In such condition, (3.21) implies that

$$
\sup _{0 \leq \tau \leq \tau_{0}} P(\tilde{k}, \tau)=0
$$

Noting that $\tau \mapsto P(\tilde{k}, \tau)$ is concave, the values $\tau^{*}$ reaching the supremum in 3.22 are fully characterized by the relation $0 \in \partial P(\tilde{k}, \tau)$, i.e.

$$
\frac{3}{c_{1}} \tilde{k} \in \partial g^{*}\left(\tau^{*}\right)
$$

As a consequence of 3.19 and (3.20), Eq. (3.23) can be rewritten as

$$
\begin{cases}\tau^{*}=\tau_{0} & \text { if } \tilde{k} \geq \frac{c_{1}}{3} \tau_{0}, \\ \tau^{*}<\tau_{0}, 3 \frac{\tilde{k}}{c_{1}}=\frac{d g^{*}}{d \tau}\left(\tau^{*}\right) & \text { if } \tilde{k}<\frac{c_{1}}{3} \tau_{0} .\end{cases}
$$

We first consider the case $\tilde{k} \geq \frac{c_{1}}{3} \tau_{0}$. In such case, the requirement 3.22 is equivalent to $P\left(\tilde{k}, \tau_{0}\right)=0$. Since $g^{*}\left(\tau_{0}\right)=3(1+\sqrt{3} / 2)$, we obtain

$$
0=(1+\sqrt{3}) \tilde{k}-\frac{\sqrt{3}}{2} \tilde{k}^{2}-c_{1}\left(1+\frac{\sqrt{3}}{2}\right)
$$

which yields

$$
\tilde{k}=\frac{1}{\sqrt{3}}\left(1+\sqrt{3}-\sqrt{1+(3+2 \sqrt{3})\left(1-c_{1}\right)}\right) .
$$

That expression holds provided it is compatible with the restriction $\tilde{k} \geq \frac{c_{1}}{3} \tau_{0}$, i.e. for $c_{1} \geq \sqrt{3} / 2$.

Let us now consider the case $\tilde{k}<\frac{c_{1}}{3} \tau_{0}$. We have $P\left(\tilde{k}, \tau^{*}\right)=0$, i.e.

$$
0=\tilde{k} \tau^{*}-\frac{\sqrt{3}}{2} \tilde{k}^{2}-\frac{c_{1}}{3} g^{*}\left(\tau^{*}\right)
$$

where $\tau^{*}<\tau_{0}$ satisfies (3.24), i.e.

$$
\frac{3}{c_{1}} \tilde{k}=\cos \theta^{*}-\sqrt{3} \sin \theta^{*}+1 .
$$

In (3.27), $\cos \theta^{*}$ and $\sin \theta^{*}$ are obtained by setting $\tau=\tau^{*}$ in (3.17). Using (3.27) and the expression (3.16) of $g^{*}$, Eq. (3.26) simplifies as

$$
0=-\frac{\sqrt{3}}{2} \tilde{k}^{2}-\frac{2}{3} c_{1}\left(\cos \theta^{*}+1\right) \sin \theta^{*}
$$


Let $a=1+\cos \theta^{*}$ and $b=-\sqrt{3} \sin \theta^{*}$. From 3.27 and 3.28 we have

$$
a+b=\frac{3}{c_{1}} \tilde{k}, a b=\frac{9}{4 c_{1}} \tilde{k}^{2} .
$$

It follows that

$$
a=\frac{3 \tilde{k}}{2 c_{1}}\left(1+\epsilon \sqrt{1-c_{1}}\right), \quad b=\frac{3 \tilde{k}}{2 c_{1}}\left(1-\epsilon \sqrt{1-c_{1}}\right)
$$

for $\epsilon \in\{-1,1\}$. Rewriting the equality $\cos ^{2} \theta^{*}+\sin ^{2} \theta^{*}=1$ in terms of $(a, b)$ leads to

$$
\tilde{k}=\frac{c_{1}\left(1+\sqrt{1-c_{1}}\right)}{2-c_{1}+\sqrt{1-c_{1}}} .
$$

The right-hand side of 3.30 is a monotonically increasing function of $c_{1}$ and is equal to $c_{1} \tau_{0} / 3$ for $c_{1}=\sqrt{3} / 2$.

In summary, the final expression of the bound $\tilde{k}$ on the effective strength of the dielectric composite is thus

$$
\tilde{k}= \begin{cases}\frac{c_{1}\left(1+\sqrt{1-c_{1}}\right)}{2-c_{1}+\sqrt{1-c_{1}}} & \text { for } c_{1} \leq \sqrt{3} / 2 \\ \frac{1}{\sqrt{3}}\left(1+\sqrt{3}-\sqrt{1+(3+2 \sqrt{3})\left(1-c_{1}\right)}\right) & \text { for } c_{1} \geq \sqrt{3} / 2\end{cases}
$$

The bound $\tilde{k}$ given by 3.31 is represented in Fig. 3, along with the Wiener bound $k_{W}$ and the linear comparison bound $k_{L C}$, respectively given by (Ponte Castañeda, 1992)

$$
k_{W}=c_{1}, k_{L C}=\frac{c_{1}}{\sqrt{2-c_{1}}} .
$$

We can observe that $\tilde{k}$ strictly improves on the linear comparison bound. The maximum improvement is about $6 \%$.

\section{Case of isotropic constituents}

In general there are substantial difficulties in evaluating the bound in (2.25). For any given $\boldsymbol{T}$ and $\alpha$, the formula $(2.25)$ indeed requires to evaluate the quantity $(W-\alpha V)^{*}(\boldsymbol{T}, \boldsymbol{x})$ defined in 2.9 , i.e. to solve a nonlinear optimization problem in $\mathbb{R}^{6}$. Since $V$ is nonconvex, local optima do not necessarily coincide with global optima and multiple solutions can exist. In addition, as illustrated in Sect. 3, the 6-dimensional vector $\boldsymbol{T}$ in $(2.25)$ as well as the scalar parameter $\alpha$ should be optimized upon in order to get relevant bounds.

We show that major simplifications occur when the constituent materials of the composite are isotropic, i.e. when the local energy function $w(\boldsymbol{e}, \boldsymbol{x})$ can be written as

$$
w(\boldsymbol{e}, \boldsymbol{x})=\phi(e, \boldsymbol{x})
$$




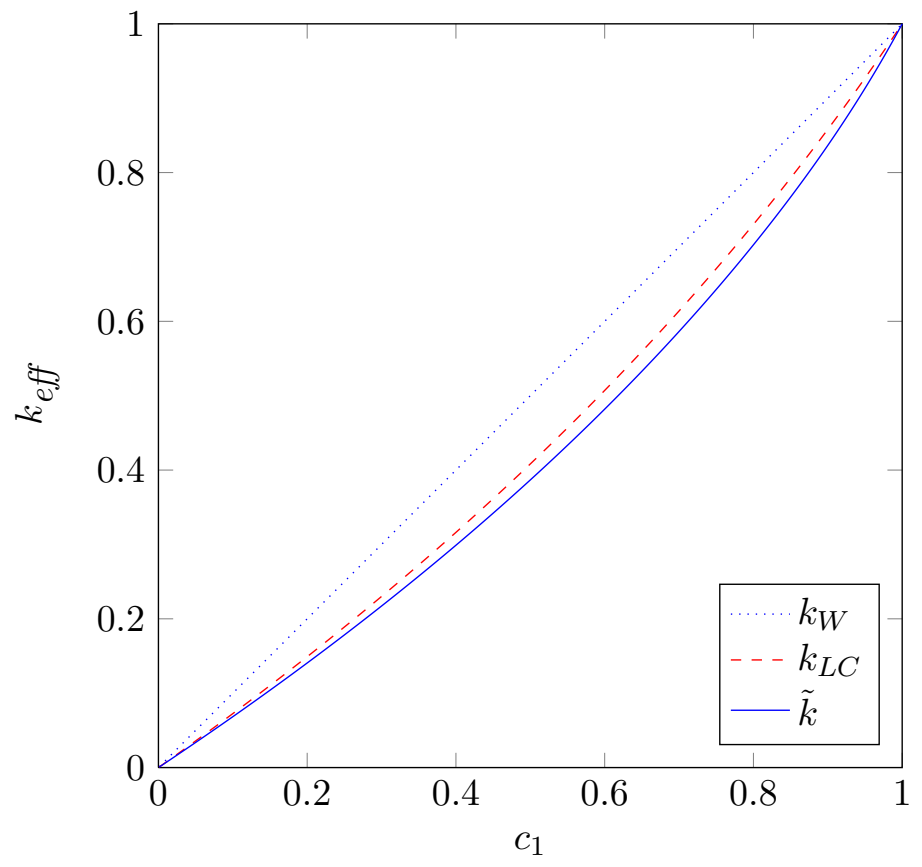

Figure 3: Upper bounds on the effective strength $k_{\text {eff }}$ of a two-phase dielectric composite: Wiener bound $k_{W}$ (dotted line), linear comparison bound $k_{L C}$ (red dashed curve), proposed bound $\tilde{k}$ (solid curve).

where $e$ denotes the norm of the vector $\boldsymbol{e}$. Under such assumption, we show in Sect. 4.2 that the values of $\boldsymbol{T}$ reaching the supremum in 2.25$)$ are necessarily parallel to the vector $\boldsymbol{N}$ introduced in 2.23 . A first consequence is that the optimization with respect to $\boldsymbol{T}$ in 2.25 is reduced from a 6 -dimensional to a 1-dimensional problem. As a second consequence, only the values taken by $(W-\alpha V)^{*}(\boldsymbol{T}, \boldsymbol{x})$ for $\boldsymbol{T}$ parallel to $\boldsymbol{N}$ are needed for evaluating the optimized bound. For such values of $\boldsymbol{T}$, the nonconvex optimization problem that defines $(W-\alpha V)^{*}(\boldsymbol{T}, \boldsymbol{x})$ can be considerably simplified, as explained in Sect. 4.3. In all the developments presented next, a central role is played by a group of linear transformations that leave $V, W$ and $\boldsymbol{N}$ invariant. Those transformations are first introduced in Sect. 4.1.

\subsection{Invariance properties}

Consider the linear transformations defined on $\mathbb{R}^{6}$ by

$$
\begin{aligned}
& \mathcal{R}_{1} \cdot\left(e_{1}, e_{2}, e_{3}\right)=\left(r\left(\frac{\pi}{3}\right) \cdot e_{2} \quad, r\left(\frac{\pi}{3}\right) \cdot e_{3} \quad, r\left(\frac{-2 \pi}{3}\right) \cdot e_{1}\right) \\
& \mathcal{R}_{2} \cdot\left(e_{1}, e_{2}, e_{3}\right)=\left(r\left(\frac{2 \pi}{3}\right) \cdot e_{3} \quad, \quad r\left(\frac{-\pi}{3}\right) \cdot e_{1}, r\left(\frac{-\pi}{3}\right) \cdot e_{2}\right) \\
& \mathcal{S}_{1} \cdot\left(e_{1}, e_{2}, e_{3}\right)=\left(s\left(\frac{-2 \pi}{3}\right) \cdot e_{1}, s\left(\frac{\pi}{3}\right) \cdot e_{3}, s\left(\frac{\pi}{3}\right) \cdot e_{2}\right) \\
& \mathcal{S}_{2} \cdot\left(e_{1}, e_{2}, e_{3}\right)=\left(s\left(\frac{2 \pi}{3}\right) \cdot e_{3} \quad, s\left(\frac{2 \pi}{3}\right) \cdot e_{2}, s\left(\frac{2 \pi}{3}\right) \cdot e_{1}\right) \\
& \mathcal{S}_{3} \cdot\left(e_{1}, e_{2}, e_{3}\right)=\left(s(\pi) \cdot e_{2} \quad, s(\pi) \cdot e_{1} \quad, s(0) \cdot e_{3} \quad\right)
\end{aligned}
$$




\begin{tabular}{c|cccccc}
$\cdot$ & $\mathcal{I}$ & $\mathcal{R}_{1}$ & $\mathcal{R}_{2}$ & $\mathcal{S}_{1}$ & $\mathcal{S}_{2}$ & $\mathcal{S}_{3}$ \\
\hline $\mathcal{I}$ & $\mathcal{I}$ & $\mathcal{R}_{1}$ & $\mathcal{R}_{2}$ & $\mathcal{S}_{1}$ & $\mathcal{S}_{2}$ & $\mathcal{S}_{3}$ \\
$\mathcal{R}_{1}$ & $\mathcal{R}_{1}$ & $\mathcal{R}_{2}$ & $\mathcal{I}$ & $\mathcal{S}_{2}$ & $\mathcal{S}_{3}$ & $\mathcal{S}_{1}$ \\
$\mathcal{R}_{2}$ & $\mathcal{R}_{2}$ & $\mathcal{I}$ & $\mathcal{R}_{1}$ & $\mathcal{S}_{3}$ & $\mathcal{S}_{1}$ & $\mathcal{S}_{2}$ \\
$\mathcal{S}_{1}$ & $\mathcal{S}_{1}$ & $\mathcal{S}_{3}$ & $\mathcal{S}_{2}$ & $\mathcal{I}$ & $\mathcal{R}_{2}$ & $\mathcal{R}_{1}$ \\
$\mathcal{S}_{2}$ & $\mathcal{S}_{2}$ & $\mathcal{S}_{1}$ & $\mathcal{S}_{3}$ & $\mathcal{R}_{1}$ & $\mathcal{I}$ & $\mathcal{R}_{2}$ \\
$\mathcal{S}_{3}$ & $\mathcal{S}_{3}$ & $\mathcal{S}_{2}$ & $\mathcal{S}_{1}$ & $\mathcal{R}_{2}$ & $\mathcal{R}_{1}$ & $\mathcal{I}$
\end{tabular}

Table 1: Group table of $\mathbb{G}$.

where

$$
\boldsymbol{r}(\alpha)=\left(\begin{array}{rr}
\cos \alpha & -\sin \alpha \\
\sin \alpha & \cos \alpha
\end{array}\right)
$$

is the two-dimensional rotation with angle $\alpha$, and

$$
\boldsymbol{s}(\alpha)=\left(\begin{array}{rr}
\cos \alpha & \sin \alpha \\
\sin \alpha & -\cos \alpha
\end{array}\right)
$$

is the two-dimensional reflection through the axis $(\cos (\alpha / 2), \sin (\alpha / 2))$. The transformations in 4.2 form a group $\mathbb{G}$ whose table is shown in Table 1 . The action of $\mathbb{G}$ on a vector $\left(\boldsymbol{e}_{\mathbf{1}}, \boldsymbol{e}_{\mathbf{2}}, \boldsymbol{e}_{\mathbf{3}}\right)$ is represented in Figure 4.

It can be verified that the function $U$ in $(2.19)$ is invariant by $\mathbb{G}$, i.e. that

$$
\mathcal{G}^{t} \cdot \mathcal{M} \cdot \mathcal{G}=\mathcal{M} \text { for all } \mathcal{G} \in \mathbb{G}
$$

where the superscript ${ }^{t}$ denotes the transpose operator. There are other linear transformations satisfying 4.3 but those in $\mathbb{G}$ have the additional property of leaving $\boldsymbol{N}$ invariant, i.e. we have

$$
\mathcal{G} \cdot \boldsymbol{N}=\boldsymbol{N} \text { for all } \mathcal{G} \in \mathbb{G} \text {. }
$$

Also observe that $W$ is invariant under $\mathbb{G}$ as the transformations in $\mathbb{G}$ have the effect of permuting the norms of the vectors $\boldsymbol{e}_{1}, \boldsymbol{e}_{2}, \boldsymbol{e}_{3}$.

We further note that all the transformations in $\mathbb{G}$ are isometries of $\mathbb{R}^{6}$, i.e. they satisfy the relation $\mathcal{G}^{t} \cdot \mathcal{G}=\mathcal{I}$. More precisely: with

- $\boldsymbol{\mathcal { R }}_{1}$ and $\boldsymbol{\mathcal { R }}_{2}$ are 'rotations', i.e. $\operatorname{det} \boldsymbol{\mathcal { R }}_{i}=1$. Their invariant $\operatorname{space}$ is $\operatorname{vect}\left(\boldsymbol{N}, \boldsymbol{N}^{\prime}\right)$

$$
\boldsymbol{N}^{\prime}=\frac{1}{2}(-\sqrt{3},-1,-\sqrt{3}, 1,0,2) .
$$

- $\mathcal{S}_{1}, \mathcal{S}_{2}$ and $\mathcal{S}_{3}$ are 'reflections', i.e. $\mathcal{S}_{i} \cdot \mathcal{S}_{i}=\mathcal{I}$. We have already noted that $\mathcal{S}_{i} \cdot \boldsymbol{N}=\boldsymbol{N}$, and it can be checked that

$$
\mathcal{S}_{i} \cdot \boldsymbol{N}^{\prime}=-\boldsymbol{N}^{\prime} \text { for } i=1,2,3 .
$$



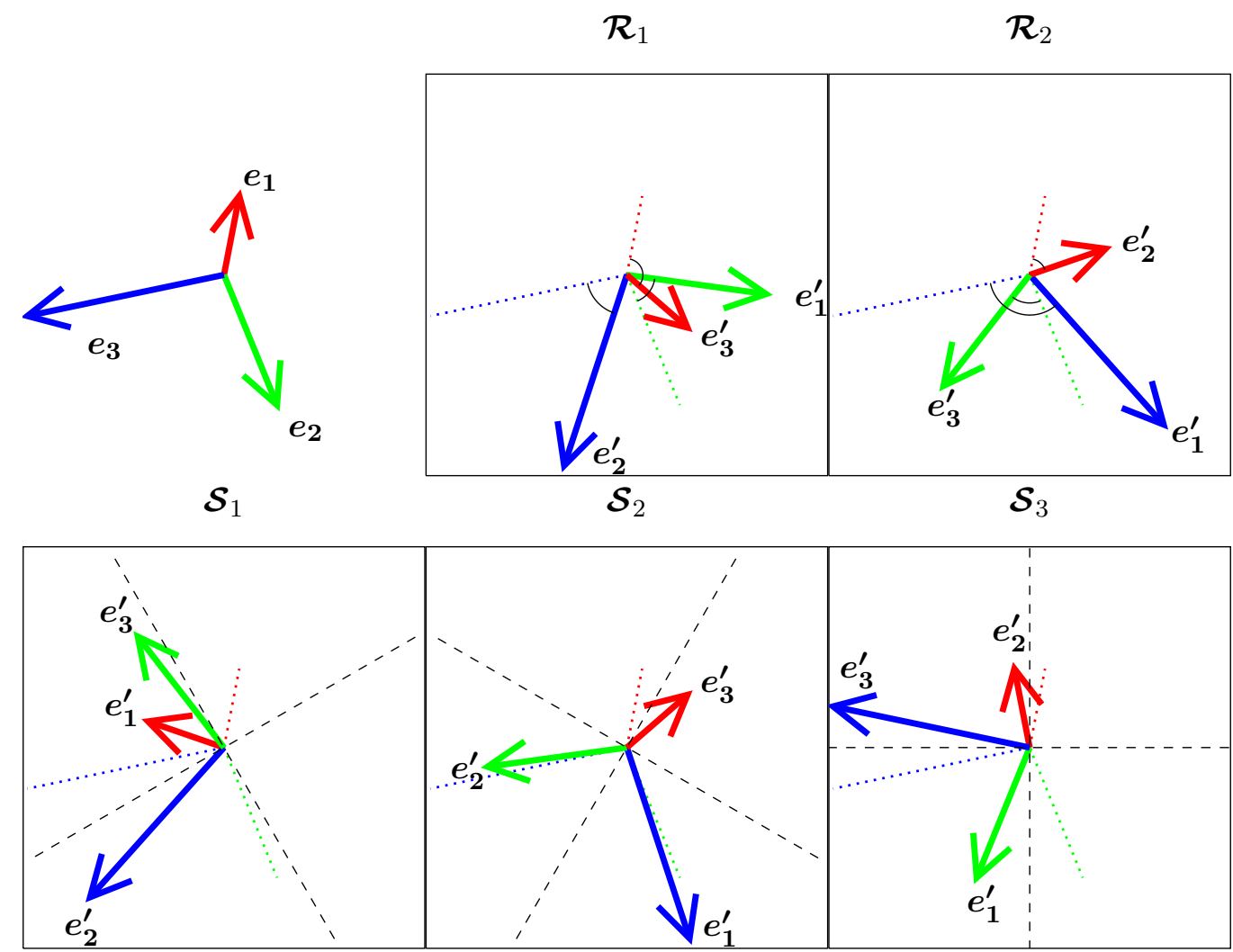

Figure 4: Action of $\mathbb{G}$ on a vector $\left(\boldsymbol{e}_{\mathbf{1}}, \boldsymbol{e}_{\mathbf{2}}, \boldsymbol{e}_{\mathbf{3}}\right)$ (shown in upper left). The vector $\left(\boldsymbol{e}_{\mathbf{1}}^{\prime}, \boldsymbol{e}_{\mathbf{2}}^{\prime}, \boldsymbol{e}_{\mathbf{3}}^{\prime}\right)$ shows the transformed of $\left(\boldsymbol{e}_{\mathbf{1}}, \boldsymbol{e}_{\mathbf{2}}, \boldsymbol{e}_{\mathbf{3}}\right)$ for each transformation in $\mathbb{G}$. The transformations $\boldsymbol{\mathcal { R }}_{i}$ involve some rotations (with angle in $\{ \pm \pi / 3, \pm 2 \pi / 3\})$ and some permutations of the vectors $\left(\boldsymbol{e}_{\mathbf{1}}, \boldsymbol{e}_{\mathbf{2}}, \boldsymbol{e}_{\mathbf{3}}\right)$. The transformations $\mathcal{S}_{i}$ involve some reflections (through axis shown as dashed lines) and some permutations of the vectors $\left(e_{1}, e_{2}, e_{3}\right)$.

For our purpose, an important observation is that

$$
\mathcal{I}+\mathcal{R}_{1}+\mathcal{R}_{2}+\mathcal{S}_{1}+\mathcal{S}_{2}+\mathcal{S}_{3}=2 \boldsymbol{N} \otimes \boldsymbol{N}
$$

We close this section with a justification of the identity (4.7). Take any arbitrary $\boldsymbol{E}$ in $\mathbb{R}^{6}$ and set $\boldsymbol{E}^{\prime}=\left(\mathcal{I}+\mathcal{R}_{1}+\mathcal{R}_{2}\right) \cdot \boldsymbol{E}$. It follows from Table 1 that

$$
\mathcal{R}_{1} \cdot \boldsymbol{E}^{\prime}=\left(\mathcal{R}_{1}+\mathcal{R}_{1}^{2}+\mathcal{R}_{1} \cdot \mathcal{R}_{2}\right) \cdot \boldsymbol{E}=\left(\mathcal{R}_{1}+\mathcal{R}_{2}+\mathcal{I}\right) \cdot \boldsymbol{E}=\boldsymbol{E}^{\prime}
$$

i.e. that $\boldsymbol{E}^{\prime}$ is invariant by $\boldsymbol{\mathcal { R }}_{1}$. Hence $\boldsymbol{E}^{\prime} \in \operatorname{vect}\left(\boldsymbol{N}, \boldsymbol{N}^{\prime}\right)$. This shows that $\mathcal{I}+\boldsymbol{\mathcal { R }}_{1}+\boldsymbol{\mathcal { R }}_{2}$ takes values in $\operatorname{vect}\left(\boldsymbol{N}, \boldsymbol{N}^{\prime}\right)$ and therefore can be written as

$$
\mathcal{I}+\mathcal{R}_{1}+\mathcal{R}_{2}=N \otimes a+N^{\prime} \otimes a^{\prime}
$$


for some $\boldsymbol{a}$ and $\boldsymbol{a}^{\prime}$ in $\mathbb{R}^{6}$. Taking the transpose of $(4.8)$ and noting from Table 1 that $\boldsymbol{\mathcal { R }}_{1}^{t}=\boldsymbol{\mathcal { R }}_{1}^{-1}=\boldsymbol{\mathcal { R }}_{2}$, we obtain

$$
\mathcal{I}+\mathcal{R}_{1}+\mathcal{R}_{2}=a \otimes N+a^{\prime} \otimes N^{\prime}
$$

Applying $\mathcal{I}+\mathcal{R}_{1}+\mathcal{R}_{2}$ to $\boldsymbol{N}$ and observing that $\boldsymbol{N} \cdot \boldsymbol{N}^{\prime}=0$ shows that $\boldsymbol{a}=\boldsymbol{N}$. Similarly, applying $\mathcal{I}+\mathcal{R}_{1}+\mathcal{R}_{2}$ to $\boldsymbol{N}^{\prime}$ gives $\boldsymbol{a}^{\prime}=\boldsymbol{N}^{\prime}$. We thus arrive at

$$
\mathcal{I}+\mathcal{R}_{1}+\mathcal{R}_{2}=N \otimes N+N^{\prime} \otimes N^{\prime}
$$

Now right-multiplying that last equation by $\mathcal{I}+\mathcal{S}_{3}$ gives

$$
\left(\mathcal{I}+\mathcal{R}_{1}+\mathcal{R}_{2}\right) \cdot\left(\mathcal{I}+\mathcal{S}_{3}\right)=\boldsymbol{N} \otimes\left(\boldsymbol{N}+\mathcal{S}_{3} \cdot \boldsymbol{N}\right)+\boldsymbol{N}^{\prime} \otimes\left(\boldsymbol{N}^{\prime}+\mathcal{S}_{3} \cdot \boldsymbol{N}^{\prime}\right)=2 \boldsymbol{N} \otimes \boldsymbol{N}
$$

where the relation $(4.6)$ has been used. Expanding $\left(\mathcal{I}+\boldsymbol{\mathcal { R }}_{1}+\boldsymbol{\mathcal { R }}_{2}\right) .\left(\boldsymbol{I}+\mathcal{S}_{3}\right)$ and using Table 1 shows that $\left(\mathcal{I}+\mathcal{R}_{1}+\mathcal{R}_{2}\right) .\left(\mathcal{I}+\mathcal{S}_{3}\right)=\mathcal{I}+\mathcal{R}_{1}+\mathcal{R}_{2}+\mathcal{S}_{1}+\mathcal{S}_{2}+\mathcal{S}_{3}$, which gives the identify 4.7 ).

\subsection{Optimization with respect to $\boldsymbol{T}$}

For a given $\alpha$, the best bound available from 2.25 is

$$
\sup _{\boldsymbol{T} \in \mathbb{R}^{6}} \frac{\bar{e}}{3} \boldsymbol{N} \cdot \boldsymbol{T}-\frac{\sqrt{3}}{2} \alpha \bar{e}^{2}-F(\boldsymbol{T})
$$

with

$$
F(\boldsymbol{T})=\frac{1}{3} \int_{\Omega}(W-\alpha V)^{*}(\boldsymbol{T}, \boldsymbol{x}) d \omega .
$$

The definition $(2.9)$ shows that $(W-\alpha V)^{*}(\boldsymbol{T}, \boldsymbol{x})$ is the supremum of linear functions in $\boldsymbol{T}$. Hence $(W-\alpha V)^{*}(\boldsymbol{T}, \boldsymbol{x})$ in convex in $\boldsymbol{T}$. Consequently, $F$ is convex and the function

$\boldsymbol{T} \longrightarrow \frac{\bar{e}}{3} \boldsymbol{N} \cdot \boldsymbol{T}-F(\boldsymbol{T})$ that appears in 4.10 is concave. It follows that the optimal values of $\boldsymbol{T}$ in 4.10 are fully characterized by the relation

$$
\frac{1}{3} \bar{e} \boldsymbol{N} \in \partial F(\boldsymbol{T}) .
$$

Similarly, for a given $\alpha$, the best bound available from $(2.26)$ is

$$
\sup _{\tau \in \mathbb{R}} \bar{e} \tau-\frac{\sqrt{3}}{2} \alpha \bar{e}^{2}-f(\tau)
$$

where

$$
f(\tau)=F(\tau \boldsymbol{N}) .
$$

The optimal values of $\tau$ in 4.13 are characterized by

$$
\bar{e} \in \partial f(\tau) .
$$


Observe from the definition of $f$ that

$$
\partial f(\tau)=\boldsymbol{N} \cdot \partial F(\tau \boldsymbol{N})
$$

Consequently, if $\boldsymbol{T}$ satisfies the optimality condition (4.12) then the scalar $\tau=\boldsymbol{T} \cdot \boldsymbol{N}$ satisfies (4.15). We show in the following that the converse is true: If $\tau$ satisfies (4.15) then the vector $\boldsymbol{T}=\tau \boldsymbol{N}$ satisfies 4.12 . This is not obvious at first sight because 4.15 is only a trace of (4.12) on a particular direction (namely, $\boldsymbol{N}$ ). The key arguments leading to the claimed result lie in the invariance properties studied in Sect. 4.1. A first observation is that the function $F$ in 4.11 is invariant by $\mathbb{G}$. Since any $\mathcal{G} \in \mathbb{G}$ verifies $\mathcal{G}^{t} \cdot \mathcal{G}=\mathcal{I}$, we have indeed

$$
\begin{aligned}
(W-\alpha V)^{*}(\boldsymbol{T}, \boldsymbol{x}) & =\sup _{\boldsymbol{E}}\{\mathcal{G} \boldsymbol{E} \cdot \mathcal{G} \boldsymbol{T}-W(\boldsymbol{E}, \boldsymbol{x})+\alpha V(\boldsymbol{E})\} \\
& =\sup _{\tilde{\boldsymbol{E}}}\left\{\tilde{\boldsymbol{E}} \cdot \mathcal{G} \boldsymbol{T}-W\left(\mathcal{G}^{-1} \tilde{\boldsymbol{E}}, \boldsymbol{x}\right)+\alpha V\left(\mathcal{G}^{-1} \tilde{\boldsymbol{E}}\right)\right\}
\end{aligned}
$$

where $\tilde{\boldsymbol{E}}=\mathcal{G} \cdot \boldsymbol{E}$. As $W$ and $V$ are invariant by $\mathbb{G}, 44.17$ gives $(W-\alpha V)^{*}(\mathcal{G T}, \boldsymbol{x})=$ $(W-\alpha V)^{*}(\boldsymbol{T}, \boldsymbol{x})$. It follows that $F$ is invariant by $\mathbb{G}$. A notable consequence is that

$$
\mathcal{G} \cdot \partial F(\boldsymbol{E}) \subset \partial F(\mathcal{G} \cdot \boldsymbol{E})
$$

for any $\boldsymbol{E}$ and $\mathcal{G} \in \mathbb{G}$. From the definition of the subdifferential, any $\boldsymbol{A} \in \partial F(\boldsymbol{E})$ indeed satisfies the distinctive property

$$
F\left(\boldsymbol{E}^{\prime}\right)-F(\boldsymbol{E}) \geq \boldsymbol{A} \cdot\left(\boldsymbol{E}^{\prime}-\boldsymbol{E}\right)
$$

where $\boldsymbol{E}^{\prime}$ is arbitrary. Since $\mathcal{G}^{t} \cdot \mathcal{G}=\mathcal{I}$ and $F$ is invariant by $\mathbb{G}, 4.19$ can be rewritten as

$$
F\left(\mathcal{G} \cdot \boldsymbol{E}^{\prime}\right)-F(\mathcal{G} \cdot \boldsymbol{E}) \geq \mathcal{G}^{t} \cdot \mathcal{G} \cdot \boldsymbol{A} \cdot\left(\boldsymbol{E}^{\prime}-\boldsymbol{E}\right)=(\mathcal{G} \cdot \boldsymbol{A}) \cdot\left(\mathcal{G} \cdot\left(\boldsymbol{E}^{\prime}-\boldsymbol{E}\right)\right)
$$

which shows that $\mathcal{G} \cdot \boldsymbol{A} \in \partial F(\mathcal{G} \cdot \boldsymbol{E})$, thus proving 4.18).

Now let $\tau$ be a scalar verifying the optimality condition (4.15). From (4.16) we have

$$
\bar{e} \in \boldsymbol{N} \cdot \partial F(\tau \boldsymbol{N})
$$

Since $\boldsymbol{N}$ is invariant by $\mathbb{G}$, using 4.18 with $\boldsymbol{E}=\tau \boldsymbol{N}$ gives

$$
\mathcal{G} \cdot \partial F(\tau \boldsymbol{N}) \subset \partial F(\tau \boldsymbol{N}) \text { for all } \mathcal{G} \in \mathbb{G} \text {. }
$$

The function $F$ being convex, $\partial F(\boldsymbol{E})$ is a convex set for all $\boldsymbol{E}$ (Rockafellar, 1970). Hence 4.21) implies that

$$
\frac{1}{6}\left(\mathcal{I}+\boldsymbol{\mathcal { R }}_{1}+\boldsymbol{\mathcal { R }}_{2}+\mathcal{S}_{1}+\mathcal{S}_{2}+\mathcal{S}_{3}\right) \cdot \partial F(\tau \boldsymbol{N}) \subset \partial F(\tau \boldsymbol{N})
$$


which using the identity (4.7) can be rewritten as

$$
\frac{1}{3}(\boldsymbol{N} \cdot \partial F(\tau \boldsymbol{N})) \boldsymbol{N} \subset \partial F(\tau \boldsymbol{N}) .
$$

In view of 4.20 , we thus obtain that

$$
\frac{1}{3} \bar{e} \boldsymbol{N} \in \partial F(\tau \boldsymbol{N})
$$

This proves that the vector $\boldsymbol{T}=\tau \boldsymbol{N}$ satisfies 4.12 and therefore reaches the supremum in 4.10 . The conclusion is that the bounds provided by 4.10 and 4.13 coincide. Consequently, the bounds 2.25 and $(2.26)$ are identical. In practice, the prescription 2.26 ) is simpler to use as it only involves 2 scalar parameters $(\tau, \alpha)$ - instead of 7 in $(2.25)$.

\subsection{Legendre transform for $\boldsymbol{T}$ parallel to $\boldsymbol{N}$}

In this section we address the evaluation of the quantity $(W-\alpha V)^{*}(\tau \boldsymbol{N}, \boldsymbol{x})$ in $(2.9)$, which is central for the evaluation of the bound (2.26). With proper rescaling, we can assume that $\alpha=1$. For a given $\boldsymbol{x},(W-V)^{*}(\tau \boldsymbol{N}, \boldsymbol{x})$ is obtained by the solving the maximization problem

$$
\sup _{\boldsymbol{E} \in \mathbb{R}^{6}} \mathcal{F}(\boldsymbol{E})
$$

where

$$
\mathcal{F}(\boldsymbol{E})=\tau \boldsymbol{E} \cdot \boldsymbol{N}-W(\boldsymbol{E}, \boldsymbol{x})+\frac{1}{2} \boldsymbol{E} \cdot \boldsymbol{M} \cdot \boldsymbol{E} .
$$

Eq. (4.23) is a nonlinear optimization problem in $\mathbb{R}^{6}$. Except in few special cases, there is no hope to solve the problem (4.23) in closed-form and one needs to resort to numerical techniques. Standard gradient algorithms are able to provide a local optimum but not necessarily a global optimum as needed for our purpose. This issue is especially sensitive as the function $\mathcal{F}$ in $(4.24)$ is non convex and expected to have multiple stationary points. Global maximization techniques are likely to overcome such difficulties, but those algorithms are much more costly than gradient algorithms. This is especially critical as the (numerical) optimization with respect to $(\alpha, \boldsymbol{T})$ in 2.26 requires numerous evaluations of $(W-\alpha V)^{*}(\tau \boldsymbol{N}, \boldsymbol{x})$. As a result, applying a global algorithm for solving (4.23) entails prohibitive computational costs when evaluating the bound in 2.26 . We show in the following that the problem can actually be reduced to a nonlinear optimization problem in $\mathbb{R}^{2}$, which makes the approach tractable.

Under the assumption 4.1) that $w(., \boldsymbol{x})$ is isotropic, the stationarity condition $\partial \mathcal{F} / \partial \boldsymbol{E}=$ 0 can be written as

$$
\left(\beta_{1} \boldsymbol{e}_{\mathbf{1}}, \beta_{2} \boldsymbol{e}_{\mathbf{2}}, \beta_{3} \boldsymbol{e}_{\mathbf{3}}\right)-\mathcal{M} \cdot \boldsymbol{E}=\tau \boldsymbol{N}
$$

where

$$
\beta_{i}=\frac{1}{e_{i}} \frac{\partial \phi}{\partial e}\left(e_{i}, \boldsymbol{x}\right)
$$


Projecting 4.25 on the canonical basis of $\mathbb{R}^{6}$ gives the 6 equations

$$
\begin{gathered}
\beta_{1} u_{1}-v_{2}-v_{3}=\frac{\tau}{2}, \\
\beta_{1} v_{1}+u_{2}+u_{3}=-\frac{\sqrt{3}}{2} \tau, \\
\beta_{2} u_{2}+v_{1}-v_{3}=-\frac{\tau}{2}, \\
\beta_{2} v_{2}-u_{1}+u_{3}=-\frac{\sqrt{3}}{2} \tau, \\
\beta_{3} u_{3}+v_{1}+v_{2}=-\tau, \\
\beta_{3} v_{3}-u_{1}-u_{2}=0 .
\end{gathered}
$$

Since $\beta_{i}$ in 4.26) is a function of $\boldsymbol{e}_{i}$, the system (4.27) is nonlinear in $\boldsymbol{e}_{i}$ and cannot be solved in closed form. However, any solution of (4.27) can be written as an explicit function of the two scalar components $u_{3}$ and $v_{3}$, as is now explained. We first rewrite the two equations $(4.27 \mathrm{~b}-\mathrm{c})$ as a linear system in $\left(v_{1}, u_{2}\right)$, namely

$$
\left(\begin{array}{ll}
\beta_{1} & 1 \\
1 & \beta_{2}
\end{array}\right)\left(\begin{array}{l}
v_{1} \\
u_{2}
\end{array}\right)=\left(\begin{array}{c}
-\frac{\sqrt{3}}{2} \tau-u_{3} \\
-\frac{\tau}{2}+v_{3}
\end{array}\right) .
$$

Provided that $\beta_{1} \beta_{2}-1 \neq 0$, the linear system 4.28 can be inverted to express $v_{1}$ and $u_{2}$ as functions of $\left(\tau, \beta_{1}, \beta_{2}, u_{3}, v_{3}\right)$. The corresponding expressions are

$$
v_{1}=\frac{1}{Y}\left(-\beta_{2} p+\frac{\tau}{2}-v_{3}\right), u_{2}=\frac{1}{Y}\left(p-\beta_{1}\left(\frac{\tau}{2}-v_{3}\right)\right) ;
$$

where

$$
Y=\beta_{1} \beta_{2}-1, p=u_{3}+\frac{\sqrt{3}}{2} \tau .
$$

Similarly, we obtain from $(4.27 \mathrm{a})$ and $(4.27 \mathrm{~d})$ that

$$
u_{1}=\frac{1}{Y}\left(\beta_{2}\left(\frac{\tau}{2}+v_{3}\right)-p\right), v_{2}=\frac{1}{Y}\left(\frac{\tau}{2}+v_{3}-\beta_{1} p\right)
$$

Substituting (4.29) and 4.31) in (4.27e) and (4.27f) gives two equations which can be written in matrix form as

$$
\left(\begin{array}{ll}
p & p \\
-\frac{\tau}{2}+v_{3} & \frac{\tau}{2}+v_{3}
\end{array}\right)\left(\begin{array}{l}
\beta_{1} \\
\beta_{2}
\end{array}\right)=\left(\begin{array}{c}
Y\left(\beta_{3} u_{3}+\tau\right)+\tau \\
\beta_{3} v_{3} Y
\end{array}\right)
$$


Inverting 4.32 with respect to $\left(\beta_{1}, \beta_{2}\right)$ gives, provided that $p \tau \neq 0$,

$$
\begin{aligned}
& \beta_{1}=\frac{\left(\frac{\tau}{2}+v_{3}\right)\left(Y\left(\beta_{3} u_{3}+\tau\right)+\tau\right)-\beta_{3} v_{3} p Y}{\tau p}, \\
& \beta_{2}=\frac{\left(\frac{\tau}{2}-v_{3}\right)\left(Y\left(\beta_{3} u_{3}+\tau\right)+\tau\right)+\beta_{3} v_{3} p Y}{\tau p} .
\end{aligned}
$$

The point of those algebraic manipulations is to express $\beta_{1}$ and $\beta_{2}$ as explicit functions of $\left(u_{3}, v_{3}\right)$. Multiplying the two equations in $(4.33)$ indeed gives a relation in which $\beta_{1}$ and $\beta_{2}$ only appear through the product $\beta_{1} \beta_{2}$. That equation can be written in terms of $Y=\beta_{1} \beta_{2}-1$ as

$$
a\left(\boldsymbol{e}_{3}\right) Y^{2}-4 b\left(\boldsymbol{e}_{3}\right) Y+4 c\left(\boldsymbol{e}_{3}\right)=0
$$

where

$$
\begin{aligned}
& a\left(\boldsymbol{e}_{3}\right)=\left(\beta_{3} u_{3}+\tau\right)^{2}-v_{3}^{2}\left(2-\sqrt{3} \beta_{3}\right)^{2}, \\
& b\left(\boldsymbol{e}_{3}\right)=p^{2}-\beta_{3} p \frac{\tau}{2}-\left(2-\sqrt{3} \beta_{3}\right)\left(\frac{1}{4} \tau^{2}-v_{3}^{2}\right), \\
& c\left(\boldsymbol{e}_{3}\right)=\frac{1}{4} \tau^{2}-p^{2}-v_{3}^{2} .
\end{aligned}
$$

To simplify the presentation, we only consider the most general situation where $a\left(\boldsymbol{e}_{3}\right) \neq 0$. Some manipulations on 4.35 show that $b^{2}\left(\boldsymbol{e}_{3}\right)-a\left(\boldsymbol{e}_{3}\right) c\left(\boldsymbol{e}_{3}\right)=p^{2} q$ where

$$
q=p^{2}+\beta_{3}\left(\tau u_{3}+\beta_{3} u_{3}^{2}+2 \sqrt{3} v_{3}^{2}-2 \beta_{3} v_{3}^{2}\right) .
$$

Therefore, if $q \geq 0$, the solutions of (4.34) are

$$
Y_{\epsilon}=2 \frac{b\left(\boldsymbol{e}_{3}\right)+\epsilon|p| \sqrt{q}}{a\left(\boldsymbol{e}_{3}\right)}
$$

where $\epsilon= \pm 1$. Setting $Y=Y_{\epsilon}$ in 4.33 gives, after some simplification,

$$
\beta_{1}=\frac{p+\epsilon \sqrt{q}+\beta_{3} v_{3}}{\tau-2 v_{3}+\beta_{3}\left(u_{3}+\sqrt{3} v_{3}\right)}, \beta_{2}=\frac{p+\epsilon \sqrt{q}-\beta_{3} v_{3}}{\tau+2 v_{3}+\beta_{3}\left(u_{3}-\sqrt{3} v_{3}\right)} .
$$

Replacing in (4.29)-(4.31), we finally arrive at

$$
\begin{cases}u_{1}=\frac{1}{2}\left(\beta_{3} v_{3}+p-\epsilon \sqrt{q}\right), & v_{1}=\frac{1}{2}\left(-\beta_{3} u_{3}-\tau+2 v_{3}-\sqrt{3} \beta_{3} v_{3}\right), \\ u_{2}=\frac{1}{2}\left(\beta_{3} v_{3}-p+\epsilon \sqrt{q}\right), & v_{2}=\frac{1}{2}\left(-\beta_{3} u_{3}-\tau-2 v_{3}+\sqrt{3} \beta_{3} v_{3}\right) .\end{cases}
$$

It can be verified that the expressions 4.36) remain valid if $p \tau=0$ or $Y=0$. Through (4.36), the components $u_{1}, v_{1}, u_{2}, v_{2}$ are all expressed as fully explicit functions of $\left(u_{3}, v_{3}\right)$ (we recall that $\beta_{3}$ is related to $\left(u_{3}, v_{3}\right)$ via 4.26$)$. 
For a vector $\boldsymbol{E}=\left(u_{1}, v_{1}, u_{2}, v_{2}, u_{3}, v_{3}\right)$ of the form (4.36), a direct calculation shows that

$$
\mathcal{F}(\boldsymbol{E})=\frac{1}{2}\left(\sqrt{3} \tau^{2}+\beta_{3} u_{3}(p+\epsilon \sqrt{q})+\sqrt{3} \beta_{3}^{2} v_{3}^{2}\right)-\sum_{i=1}^{3} w\left(\sqrt{u_{i}^{2}+v_{i}^{2}}, \boldsymbol{x}\right) .
$$

The analysis so far shows that stationary points of $\mathcal{F}$ are necessary of the form 4.36 and therefore can be parameterized by $\left(u_{3}, v_{3}\right)$. In order to evaluate $\sup _{\mathbb{R}^{6}} \mathcal{F}$, we thus only need to look at the values taken by $\mathcal{F}$ over $\boldsymbol{E}$ of the form (4.36), i.e. we are left with solving the two optimization problems $\sup _{\left(u_{3}, v_{3}\right)} \mathcal{F}_{+}\left(u_{3}, v_{3}\right)$ and $\sup _{\left(u_{3}, v_{3}\right)} \mathcal{F}_{-}\left(u_{3}, v_{3}\right)$ where

$$
\mathcal{F}_{ \pm}\left(u_{3}, v_{3}\right)=\frac{1}{2}\left(\sqrt{3} \tau^{2}+\beta_{3} u_{3}(p \pm \sqrt{q})+\sqrt{3} \beta_{3}^{2} v_{3}^{2}\right)-\sum_{i=1}^{3} w\left(\sqrt{u_{i}^{2}+v_{i}^{2}}, \boldsymbol{x}\right) .
$$

At this point the optimization problem 4.23 in $\mathbb{R}^{6}$ has been reduced to two optimization problems in $\mathbb{R}^{2}$. Observe from 4.36 that $u_{2}+u_{3}-u_{1}+\sqrt{3} \tau / 2=\epsilon \sqrt{q}$. Hence maximizing $\mathcal{F}_{+}$amounts to look for solutions to 4.23 that lie in the half-space $\mathcal{H}_{+}$of $\mathbb{R}^{6}$ defined by the equation $u_{2}+u_{3}-u_{1}+\sqrt{3} \tau / 2 \geq 0$. Similarly, maximizing $\mathcal{F}_{-}$amounts to look for solutions in the complementary half-space $\mathcal{H}_{-}$defined by the equation $u_{2}+u_{3}-u_{1}+\sqrt{3} \tau / 2 \leq 0$.

Using invariance properties, the problem can further be simplified if $w$ is strongly convex, in the sense that the function $\phi$ in 4.1 is convex in $e^{2}$. Observe indeed that the function $\mathcal{F}$ in $(4.24)$ is invariant by the group of transformations $\mathbb{G}$ introduced in Sect. 4.1. Therefore, if $\boldsymbol{E}^{*}$ reaches the supremum in $(4.23)$ then so does $\boldsymbol{\mathcal { R }}_{1} \boldsymbol{E}^{*}$ and $\boldsymbol{\mathcal { R }}_{2} \boldsymbol{E}^{*}$. Assuming that $w$ is strongly convex, it can be proved that $\boldsymbol{E}^{*}, \boldsymbol{\mathcal { R }}_{1} \boldsymbol{E}^{*}, \boldsymbol{\mathcal { R }}_{2} \boldsymbol{E}^{*}$ cannot be in the same half-space $\mathcal{H}_{ \pm}$: if (say) $\boldsymbol{E}^{*} \in \mathcal{H}_{+}$, then either $\boldsymbol{\mathcal { R }}_{1} \boldsymbol{E}^{*}$ or $\boldsymbol{\mathcal { R }}_{2} \boldsymbol{E}^{*}$ is in $\mathcal{H}_{-}$. This implies that

$$
\sup _{\boldsymbol{E} \in \mathbb{R}^{6}} \mathcal{F}(\boldsymbol{E})=\sup _{\left(u_{3}, v_{3}\right)} \mathcal{F}_{+}\left(u_{3}, v_{3}\right)=\sup _{\left(u_{3}, v_{3}\right)} \mathcal{F}_{-}\left(u_{3}, v_{3}\right)
$$

Hence $\sup _{\boldsymbol{E} \in \mathbb{R}^{6}} \mathcal{F}(\boldsymbol{E})$ can be obtained by solving indifferently one of the two optimization problems $\sup _{\left(u_{3}, v_{3}\right)} \mathcal{F}_{+}$or $\sup _{\left(u_{3}, v_{3}\right)} \mathcal{F}_{-}$. In most cases, numerical computations are needed to carry out that optimization.

\section{Results for power-law composites}

In this section are presented some results for two-phase isotropic composites governed by power-law energy functions. The energy function $w_{i}$ in phase $i(i=1,2)$ is taken as

$$
w_{i}(\boldsymbol{e})=\frac{\sigma_{i}}{n+1} e^{n+1}
$$

where $n>0$ is a nonlinearity index and $\sigma_{i}>0$ is a nonlinear conductivity parameter. The limiting case $\sigma_{i} \rightarrow \infty$ corresponds to a perfect conductor. The case $n=1$ corresponds to a linear behavior, whereas the limiting case $n \rightarrow \infty$ corresponds to a perfect dielectric as 
considered in Sect. 3.2. By Legendre duality, the complementary energy functions $w_{i}^{*}$ is given by

$$
w_{i}^{*}(\boldsymbol{j})=\frac{\chi_{i}}{m+1} j^{m+1}
$$

where $m=1 / n$ and $\chi_{i}=\sigma_{i}^{-1 / n}$ is the nonlinear resistivity in phase $i$. In $5.2, j$ denotes the norm of the vector $\boldsymbol{j}$.

The index $n$ in (5.1) is assumed to be the same for both phases. In such case, one can show that the effective energy $w_{\text {eff }}$ of the composite is of the form

$$
w_{\text {eff }}(\overline{\boldsymbol{e}})=\frac{\sigma_{\text {eff }}}{n+1} \bar{e}^{n+1}
$$

where $\sigma_{\text {eff }}$ is the effective nonlinear conductivity. Correspondingly, the complementary effective energy $w_{\text {eff }}^{*}$ is given by

$$
w_{\text {eff }}^{*}(\bar{\jmath})=\frac{\chi_{\text {eff }}}{m+1} \bar{\jmath}^{m+1}
$$

where the effective resistivity $\chi_{\text {eff }}$ is related to the effective conductivity $\sigma_{\text {eff }}$ by $\chi_{\text {eff }}=$ $\sigma_{\text {eff }}^{-1 / n}$.

Bounding the energy function $w_{\text {eff }}$ (or the complementary energy function $w_{\text {eff }}^{*}$ ) thus amounts to bounding the nonlinear conductivity $\sigma_{\text {eff }}$ (or the nonlinear resistivity $\chi_{\text {eff }}$ ). For $n \geq 1$, the proposed methodology delivers a lower bound $\tilde{\sigma}$ on $\sigma_{\text {eff }}$ that is given by

$$
\frac{\tilde{\sigma}}{n+1}=\sup _{\alpha \in \mathbb{R}, \tau \in \mathbb{R}}\left\{\tau-\frac{\sqrt{3}}{2} \alpha-\frac{1}{3} \sum_{i=1}^{2} c_{i}\left(W_{i}-\alpha V\right)^{*}(\tau \boldsymbol{N})\right\}
$$

where $\left(W_{i}-\alpha V\right)^{*}$ is defined as in (3.12). The normalized bound $\tilde{\sigma} / \sigma_{1}$ is a function of the volume fraction $c_{i}$, of the ratio $\sigma_{2} / \sigma_{1}$ and of the nonlinearity index $n$. The (normalized) linear comparison bound $\sigma_{L C} / \sigma_{1}$ depends on the same parameters and is used as a reference in the following. The linear comparison bound $\sigma_{L C}$ can be obtained using either the Talbot and Willis (1985) approach, or by combining the variational approach of Ponte Castañeda (1991) with the Hashin-Shtrikman linear bound (3.6).

We first consider the case where phase 2 is a perfect conductor. In such case, the contrast is infinite and the bounds only depend on the two parameters $\left(c_{1}, n\right)$. The case of finite contrast is next addressed in Sect. 5.2.

\subsection{Perfectly conducting inclusions in a power-law matrix}

Consider a composite mixing a power-law matrix (phase 1) with a perfectly conducting medium (phase 2). The energy $w_{2}$ in the perfectly conducting phase 2 is given by (3.8). 
The exact expression of the linear comparison bound $\sigma_{L C}$ can be obtained in closed-form (Ponte Castañeda, 1992) and reads as

$$
\frac{\sigma_{L C}}{\sigma_{1}}=\frac{\left(2-c_{1}\right)^{\frac{n+1}{2}}}{c_{1}^{n}} .
$$

The corresponding upper bound $\chi_{L C}$ on the effective resistivity is given by $\chi_{L C} / \chi_{1}=$ $c_{1} /\left(2-c_{1}\right)^{\frac{n+1}{2 n}}$.

Since $\left(W_{2}-\alpha V\right)^{*}(\tau \boldsymbol{N})=0$, the bound (5.4) simplifies as

$$
\frac{\tilde{\sigma}}{n+1}=\sup _{\alpha \in \mathbb{R}, \tau \in \mathbb{R}}\left\{\tau-\frac{\sqrt{3}}{2} \alpha-\frac{c_{1}}{3}\left(W_{1}-\alpha V\right)^{*}(\tau \boldsymbol{N})\right\} .
$$

The bound $\tilde{\sigma}$ in (5.6) is estimated numerically. As mentioned earlier, the most sensitive issue lies in the calculation of the Legendre transform $\left(W_{1}-\alpha V\right)^{*}(\tau \boldsymbol{N})$. Following the developments of Sect. 4, the value of $\left(W_{1}-\alpha V\right)^{*}(\tau \boldsymbol{N})$ is obtained by maximizing the function $\mathcal{F}_{+}$in Eq. 4.37) over $\mathbb{R}^{2}$. Because of the growth behavior of $w_{1}$, the search for the supremum of $\mathcal{F}_{+}$can be restricted to a disk of prescribed radius $R$ (see Appendix $\mathrm{B}$ ), which is helpful for the numerical optimization.

As an illustration, the function $\mathcal{F}_{+}$is plotted in Fig. 5 in the particular example $n=3$, $\sigma_{1}=1, \tau=9.2$. The symmetry of the function $\mathcal{F}_{+}$with respect to the axis $v_{3}=0$ is a direct consequence of the expressions (4.36) and (4.37). As can be observed in Fig. 5 , the function $\mathcal{F}_{+}$has several local maxima so that special care must be taken when using a gradient algorithm for finding a global maximum: Depending on the initial guess that is used, a gradient algorithm may converge to a local maximum that does not correspond to a global maximum. To overcome such difficulty, the numerical strategy that we have used consists in discretizing the disk of radius $R$ in a large $(\simeq 10000)$ number of sample points, and looking at the sample point with the largest value of $\mathcal{F}_{+}$. That particular sample is next used as the initial guess in a gradient algorithm. For the case depicted in Fig. 5, the global maximum is indicated by the red arrows and is reached at two values of $\left(u_{3}, v_{3}\right)$.

Some contour plots of the function $f(\tau, \alpha)=\tau-\frac{\sqrt{3}}{2} \alpha-\frac{c_{1}}{3}\left(W_{1}-\alpha V\right)^{*}(\tau \boldsymbol{N})$ are represented in Fig. 6 for a volume fraction $c_{1}$ set to 0.7 . Maximizing the function $f$ with respect to the two scalar parameters $(\tau, \alpha)$ gives the best bound available from the proposed procedure - see Eq. (5.6). The function $f$ is convex in $(\tau, \alpha)$ so there is no substantial difficulty in estimating numerically its maximum. In the example considered, the maximum is reached for $(\tau, \alpha) \simeq(17.9,1.94)$. The corresponding bound on $\sigma_{\text {eff }}$ is approximatively equal to 5.58 .

\subsubsection{Influence of the nonlinearity index $n$}

The bound $\tilde{\sigma} / \sigma_{1}$ is plotted in Fig. 7 (left) as a function of the nonlinearity index $n$, for a volume fraction $c_{1}=0.7$. The bound $\tilde{\sigma}$ is obtained by solving 5.6 numerically using the procedure exposed previously. The linear comparison bound $\sigma_{L C}$ is shown in dashed lines. 


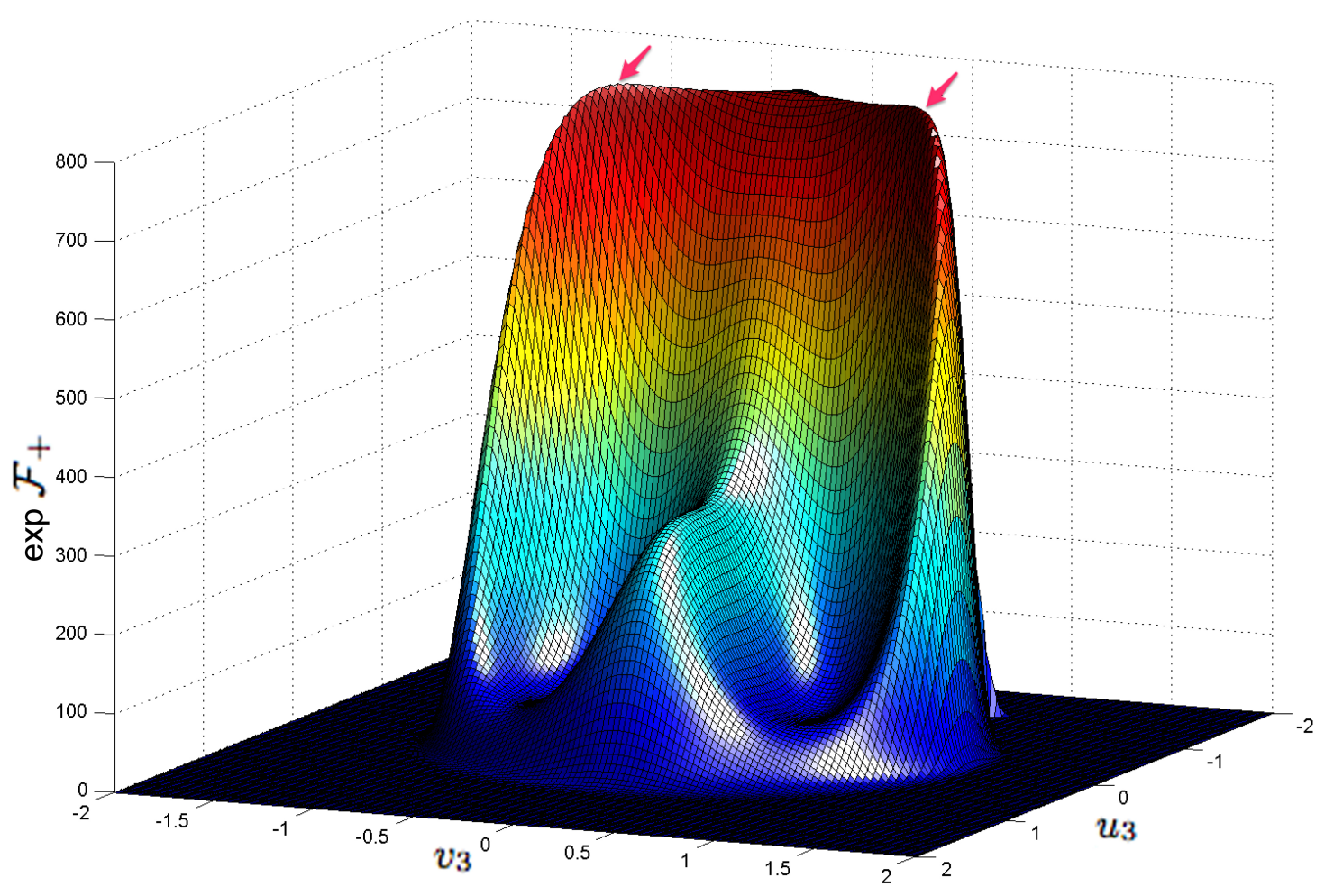

Figure 5: Plot of $\mathcal{F}_{+}\left(u_{3}, v_{3}\right)$ for $n=3, \sigma_{1}=1, \tau=9.2$.

As can be observed in Fig. 7(left) the bound $\tilde{\sigma}$ strictly improves on $\sigma_{L C}$ - except in the special case $n=1$ where both $\tilde{\sigma}$ and $\sigma_{L C}$ reduce to the linear Hashin-Shtrikman bound (3.6). The relative improvement $\left(\tilde{\sigma}-\sigma_{L C}\right) / \sigma_{L C}$ is found to increase with $n$ and reaches $13 \%$ for $n=3$. In Fig. 7 (right) is plotted the corresponding upper bound $\tilde{\chi}$ on the effective resistivity $\chi_{\text {eff }}$ as obtained through the relation $\tilde{\chi} / \chi_{1}=\left(\sigma_{1} / \tilde{\sigma}\right)^{1 / n}$. In terms of resistivity, the relative improvement on the comparison bound remains small $\left(\left(\chi_{L C}-\tilde{\chi}\right) / \chi_{L C} \simeq 4 \%\right.$ for $n=3)$.

The bound $\tilde{k}$ obtained in Sect. 3.2 can be recovered from the asymptotic behavior of $\tilde{\chi}$ as $n \rightarrow \infty$. In the limit $n \rightarrow \infty$, the power-law energy $w_{1}$ in $(5.1)$ indeed reduces to the energy (3.7) of a perfect dielectric with unit strength so that the composite behaves as in Sect. 3.2 From the expressions 3.10$)$ and 5.3 of the effective complementary energy, we thus expect that $\chi_{\text {eff }} \underset{n \rightarrow+\infty}{\longrightarrow} k_{\text {eff }}$ and similarly that

$$
\tilde{\chi} \underset{n \rightarrow+\infty}{\longrightarrow} \tilde{k}
$$

where the expression of $\tilde{k}$ is given in 3.31. For $c_{1}=0.7$, Eq. 3.31 gives $\tilde{k}=0.5865$. 


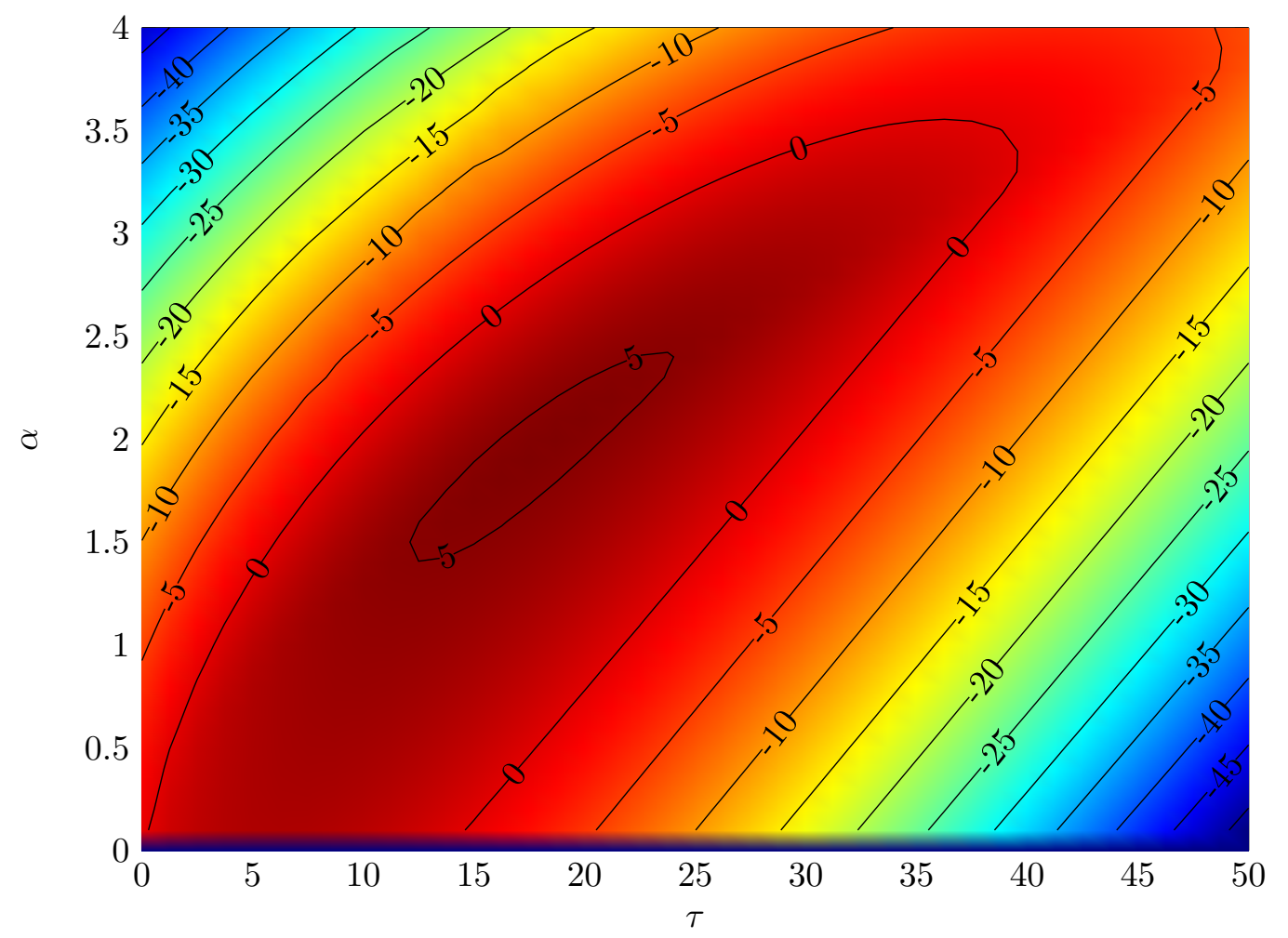

Figure 6: Contour plots of $f(\tau, \alpha)$ for $c_{1}=0.7, n=3, \sigma_{1}=1$.

As can be observed in Fig. 7(right), the convergence of $\tilde{\chi}$ towards the asymptotic value $\tilde{k}$ (shown in dotted line) is relatively slow. The linear comparison bound displays a similar behavior.

\subsubsection{Influence of $c_{1}$}

The influence of the volume fraction $c_{1}$ on the bounds is illustrated in Fig. 8(left) in the case $n=3$. The results are presented in terms of resistivity. For any given $n>1$, the relative improvement $\left(\chi_{L C}-\tilde{\chi}\right) / \chi_{L C}$ decreases with the volume fraction $c_{1}$ of the matrix, as shown in Fig. 8 (right). Values above $5 \%$ of the relative improvement $\left(\chi_{L C}-\tilde{\chi}\right) / \chi_{L C}$ can be reached as soon as $n \geq 2.5$. Correspondingly, the relative improvement $\left(\tilde{\sigma}-\sigma_{L C}\right) / \sigma_{L C}$ can exceed $10 \%$ for $n \geq 2.5$.

\subsection{Influence of the contrast}

We now consider the situation where the nonlinear conductivities $\sigma_{1}$ and $\sigma_{2}$ take finite, non-zero values. Without loss of generality, we can label the phases so that $\sigma_{2} / \sigma_{1}>1$. The bound $\tilde{\sigma}$ in (5.4) is estimated using a numerical procedure similar to that presented in 

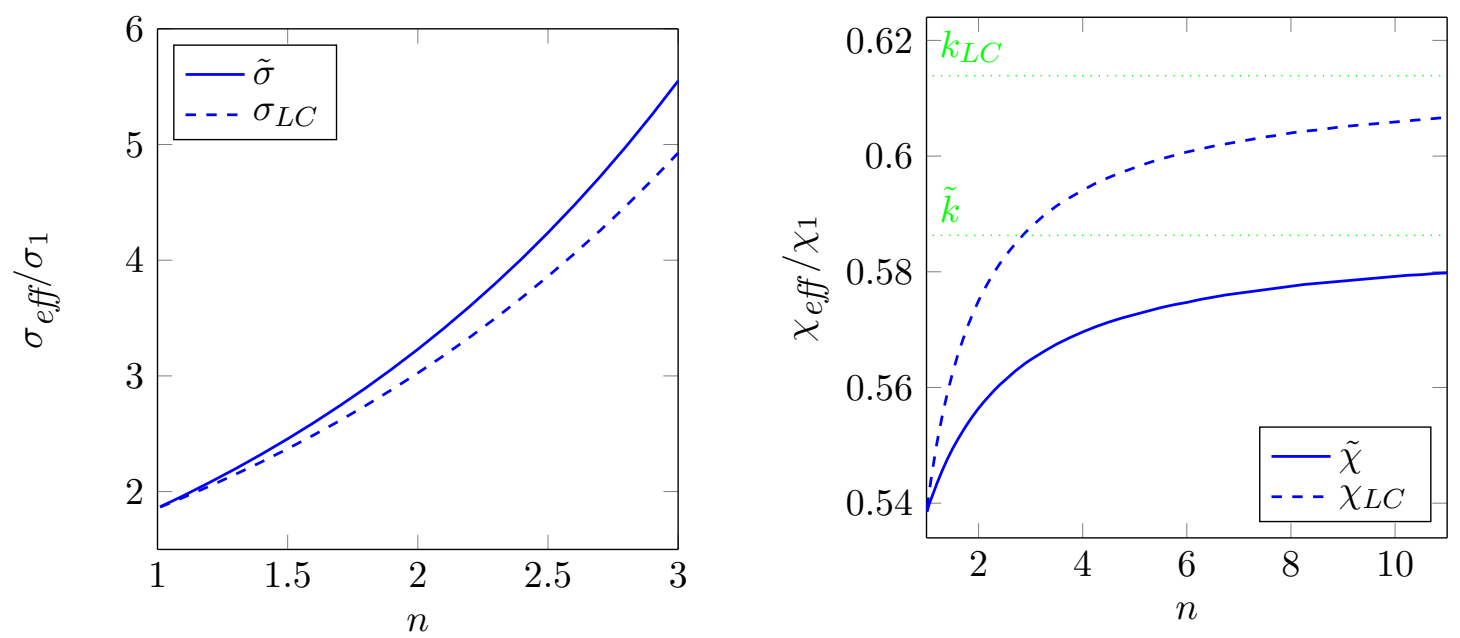

Figure 7: Bounds on the effective properties of a power-law composite, as a function of the nonlinearity index $n$. Case of infinite contrast, with $c_{1}=0.7$. Solid line: proposed bound. Dashed line: linear comparison bound.
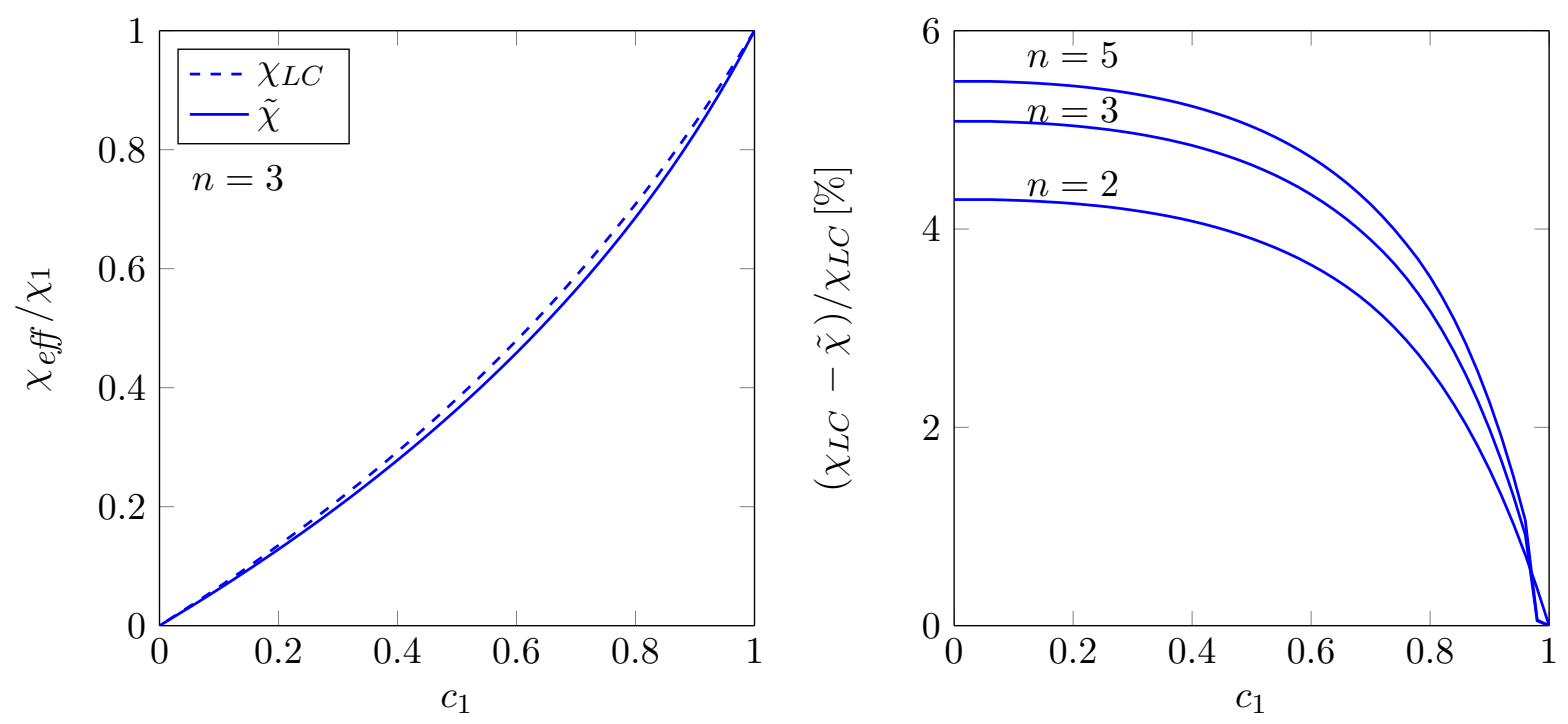

Figure 8: Left: Upper bounds on the effective resistivity of a power-law composite, as a function of the volume fraction $c_{1}$. Case of infinite contrast, with $n=3$. Solid line: proposed bound. Dashed line: linear comparison bound. Right: relative improvement as a function of the volume fraction $c_{1}$ for $n=2,3,5$.

Sect. 5.1. Contrary to the case considered in Sect5.1, there is no analytical expression of the linear comparison bound $\sigma_{L C}$ so the latter has to be evaluated numerically as well. The bounds obtained are shown in Fig. 9 as functions of the contrast ratio, in the case $n=3$. 
In a way similar to Fig. 8 , the results in Fig. 9 are presented in terms of the resistivity $\chi=\sigma^{-1 / n}$. For the highest contrast considered in the calculations $\left(\sigma_{1} / \sigma_{2}=100\right.$ i.e. $\chi_{2} / \chi_{1} \simeq 0.2$ ), the relative improvement $\left(\chi_{L C}-\tilde{\chi}\right) / \chi_{L C}$ is in the range $2-3 \%$ (for volume fractions $c_{1}$ about 0.5$)$. In terms of conductivity, the corresponding relative improvement $\left(\tilde{\sigma}-\sigma_{L C}\right) / \sigma_{L C}$ is the range $7-10 \%$.

At small contrast (i.e. $\sigma_{2}=\sigma_{1}(1+r)$ with $\left.r \ll 1\right)$, the effective conductivity $\sigma_{\text {eff }}$ is known to admit the expansion (Blumenfeld and Bergman, 1989, 1991)

$$
\frac{\sigma_{e f f}}{\sigma_{1}}=1+c_{2} r-\frac{n+1}{2(n+\sqrt{n})} c_{1} c_{2} r^{2}+O\left(r^{3}\right)
$$

The small-contrast expansion of the linear comparison bound is given by (Ponte Castañeda et al. 1992)

$$
\frac{\sigma_{L C}}{\sigma_{1}}=1+c_{2} r-\frac{1}{2} c_{1} c_{2} r^{2}+O\left(r^{3}\right)
$$

As can observed on those expressions, the linear comparison is exact only to first-order in contrast (except in the special cases $n=1$ and $n=\infty$ ). It can be verified that

$$
\frac{\tilde{\sigma}}{\sigma_{1}}=1+c_{2} r+O\left(r^{2}\right)
$$

i.e. the bound $\tilde{\sigma}$ is exact to first-order in contrast - just as the linear comparison bound $\sigma_{L C}$. Although we have not determined the second-order expansion of the bound $\tilde{\sigma}$ (the calculations proved to be quite cumbersome), the numerical results shown in Fig. 9 suggest that the bound $\tilde{\sigma}$ is not exact to second-order in contrast. For instance, in the case $n=3$, $c_{1}=0.5$, the expression $(5.7)$ gives

$$
\lim _{r \rightarrow 0} \frac{\sigma_{e f f} / \sigma_{1}-1-c_{2} r}{r^{2}}=-\frac{n+1}{2(n+\sqrt{n})} c_{1} c_{2} \simeq-0.106 .
$$

The numerical results give

$$
\lim _{r \rightarrow 0} \frac{\tilde{\sigma} / \sigma_{1}-1-c_{2} r}{r^{2}} \simeq-0.113
$$

which does not match with the value in (5.9). As a comparison, the expression (5.8) gives

$$
\lim _{r \rightarrow 0} \frac{\sigma_{L C} / \sigma_{1}-1-c_{2} r}{r^{2}}=-0.125
$$

The proposed bound gives a second-order expansion that is closer to $(5.9)$ but a significant gap remains. 

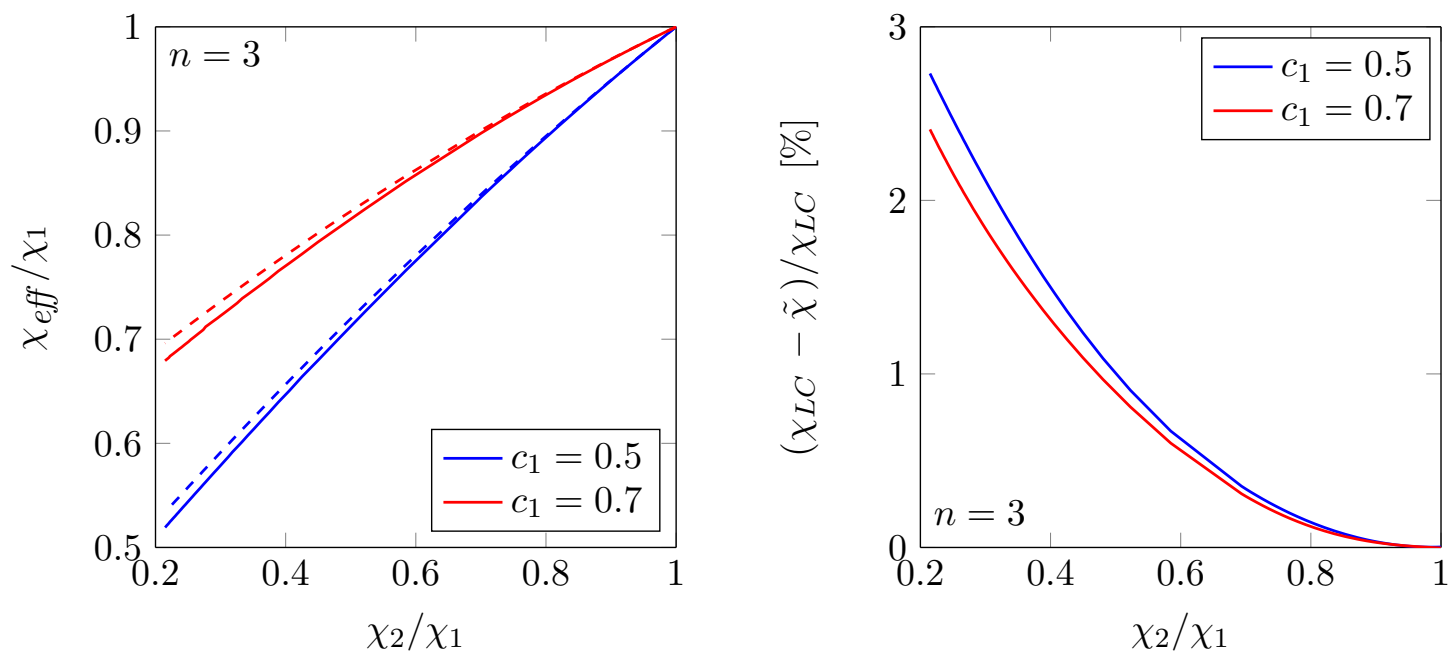

Figure 9: Left: Upper bounds on the effective resistivity of a power-law composite, as a function of the contrast ratio. Solid line: proposed bound. Dashed line: linear comparison bound. Right: relative improvement as a function of the contrast ratio.

\section{Bounds on the effective complementary energy}

The methodology is now adapted to bound the effective complementary energy $w_{\text {eff }}^{*}$ from below (which is equivalent to bounding $w_{\text {eff }}$ from above). In the isotropic case, $w_{\text {eff }}^{*}$ only depends on $\bar{\jmath}$ through its norm $\bar{\jmath}$, i.e. we have

$$
w_{\text {eff }}^{*}(\bar{\jmath})=\phi_{\text {eff }}^{*}(\bar{\jmath})
$$

for some function $\phi_{\text {eff }}^{*}$. Setting $\boldsymbol{J}=\left(\boldsymbol{j}_{1}, \cdots, \boldsymbol{j}_{n}\right)$, we consider the extended complementary energy

$$
W^{*}(\boldsymbol{J}, \boldsymbol{x})=\sum_{i=1}^{n} w^{*}\left(\boldsymbol{j}_{i}, \boldsymbol{x}\right) .
$$

Let $\overline{\boldsymbol{J}}=\left(\overline{\boldsymbol{\jmath}}_{1}, \cdots, \overline{\boldsymbol{\jmath}}_{n}\right)$ be such that $\bar{\jmath}_{1}=\cdots=\bar{\jmath}_{n}=\bar{\jmath}$. Reproducing the arguments of Sect. 2 leads to the lower bound

$$
\phi_{\text {eff }}^{*}(\bar{\jmath}) \geq \frac{1}{n}\left(\overline{\boldsymbol{J}} \cdot \boldsymbol{T}+\tilde{U}(\overline{\boldsymbol{J}})-\int_{\Omega}\left(W^{*}-\tilde{U}\right)^{*}(\boldsymbol{T}, \boldsymbol{x}) d \omega\right)
$$

where $\tilde{U}$ satisfies 2

$$
\int_{\Omega} \tilde{U}(\boldsymbol{J}) d \omega \geq \tilde{U}(\overline{\boldsymbol{J}}) \text { for any } \boldsymbol{J} \in \mathcal{S}(\overline{\boldsymbol{J}})
$$

\footnotetext{
${ }^{2}$ The distinctive property 6.2 enters into the framework of $\mathcal{A}$-quasiconvexity (Fonseca and Müller 1999 ) and can be interpreted as quasiconvexity in divergence-free fields. To avoid any confusion, use of the word 'quasiconvexity' is restricted in this paper to the standard definition 2.12 .
} 
with

$$
\mathcal{S}(\overline{\boldsymbol{J}})=\left\{\left(\boldsymbol{j}_{1}, \cdots, \boldsymbol{j}_{n}\right): \operatorname{div} \boldsymbol{j}_{i}=0 \text { in } \Omega, \boldsymbol{j}_{i}(\boldsymbol{x}) \cdot \boldsymbol{n}=\overline{\boldsymbol{j}}_{i} \cdot \boldsymbol{n} \text { on } \partial \Omega\right\}
$$

For two-dimensional problems, potentials $\tilde{U}$ that satisfy 6.2 can easily be generated from quasiconvex potential $U$, i.e. potentials that satisfy 2.12 . For any given potential $U$ defined in $\mathbb{R}^{2 n}$, considered indeed the 'rotated' potential $U_{\perp}$ defined by

$$
U_{\perp}(\boldsymbol{J})=U\left(\boldsymbol{r}_{\perp} \boldsymbol{J}\right)
$$

where $\boldsymbol{r}_{\perp} \boldsymbol{J}=\left(\boldsymbol{r}_{\perp} \boldsymbol{j}_{1}, \cdots, \boldsymbol{r}_{\perp} \boldsymbol{j}_{n}\right)$ and $\boldsymbol{r}_{\perp}$ is the two-dimensional rotation with angle $\pi / 2$. For two-dimensional fields, we have

$$
\boldsymbol{j} \in \mathcal{S}(\overline{\boldsymbol{\jmath}}) \Longleftrightarrow \boldsymbol{r}_{\perp} \boldsymbol{j} \in \mathcal{K}\left(\boldsymbol{r}_{\perp} \overline{\boldsymbol{\jmath}}\right) .
$$

Let us briefly justify the property (6.3). It is well-known (and easy to verify) that $\boldsymbol{r}_{\perp}$ maps divergence-free fields to curl-free fields. For any $\boldsymbol{j} \in \mathcal{S}(\overline{\boldsymbol{\jmath}})$, the rotated field $\boldsymbol{r}_{\perp} \boldsymbol{j}$ can thus be written as $\boldsymbol{r}_{\perp} \boldsymbol{j}=\nabla u$ for some function $u$. From the boundary condition $\boldsymbol{j} \cdot \boldsymbol{n}=\overline{\boldsymbol{j}} \cdot \boldsymbol{n}$, we obtain that $\left(\nabla u-\boldsymbol{r}_{\perp} \overline{\boldsymbol{J}}\right) \wedge \boldsymbol{n}=0$ on $\partial \Omega$, i.e. that $u=\left(\boldsymbol{r}_{\perp} \overline{\boldsymbol{J}}\right) \cdot \boldsymbol{x}$ on $\partial \Omega$ (up to some constant that can be taken as 0). This shows that $\boldsymbol{r}_{\perp} \boldsymbol{j} \in \mathcal{K}\left(\boldsymbol{r}_{\perp} \overline{\boldsymbol{j}}\right)$. We note that the relation (6.3) also holds for extended fields, i.e.

$$
\boldsymbol{J} \in \mathcal{S}(\overline{\boldsymbol{J}}) \Longleftrightarrow \boldsymbol{r}_{\perp} \boldsymbol{J} \in \mathcal{K}\left(\boldsymbol{r}_{\perp} \overline{\boldsymbol{J}}\right) .
$$

A notable consequence of 6.4 is that $U_{\perp}$ satisfies 6.2 whenever $U$ is quasiconvex. For any $\boldsymbol{J} \in \mathcal{S}(\overline{\boldsymbol{J}})$, we have indeed

$$
\int_{\Omega} U_{\perp}(\boldsymbol{J}) d \omega=\int_{\Omega} U\left(\boldsymbol{r}_{\perp} \boldsymbol{J}\right) d \omega \geq U\left(\boldsymbol{r}_{\perp} \overline{\boldsymbol{J}}\right)=U_{\perp}(\overline{\boldsymbol{J}})
$$

where the inequality comes from the quasiconvexity of $U$ together with the fact that $\boldsymbol{r}_{\perp} \boldsymbol{J} \in$ $\mathcal{K}\left(\boldsymbol{r}_{\perp} \overline{\boldsymbol{J}}\right)$.

We now specialize the above relations to $n=3$ and to quasiconvex potentials $U$ of the form (2.19). Since the two-dimensional determinant is invariant by $\boldsymbol{r}_{\perp}$, the quasiconvex function $V$ in 2.20) satisfies $V_{\perp}=V$. Taking $\overline{\boldsymbol{J}}=\bar{\jmath} \boldsymbol{N}$ and $\tilde{U}=\alpha V$ in (6.1), we obtain the following lower bound on the complementary effective energy:

$$
\phi_{e f f}^{*}(\bar{\jmath}) \geq \sup _{\alpha \in \mathbb{R}, \tau \in \mathbb{R}}\left\{\bar{\jmath} \tau-\frac{\sqrt{3}}{2} \alpha \bar{\jmath}^{2}-\frac{1}{3} \int_{\Omega}\left(W^{*}-\alpha V\right)^{*}(\tau \boldsymbol{N}, \boldsymbol{x}) d \omega\right\} .
$$

Observe that (6.5) is formally identical to 2.26$)$. Consequently, all the developments of Sect. 4 remain valid by making the formal substitutions $W \mapsto W^{*}$ and $\boldsymbol{E} \mapsto \boldsymbol{J}$. 


\subsection{Perfectly insulating inclusions in a power-law matrix}

As an illustration, consider a power-law matrix (phase 1) with perfectly insulating inclusions (phase 2). The energy $w_{1}$ is of the form (5.1). The energy $w_{2}(\boldsymbol{e})$ is null for all $\boldsymbol{e}$. By Legendre duality, the complementary energy function $w_{2}^{*}$ is given by

$$
w_{2}^{*}(\boldsymbol{j})= \begin{cases}0 & \text { if } j=0 \\ +\infty & \text { otherwise }\end{cases}
$$

The effective complementary energy $w_{\text {eff }}^{*}$ is of the form 5.3 . The formula 6.5 gives the inequality $\tilde{\chi} \leq \chi_{\text {eff }}$ where $\chi_{\text {eff }}$ is the effective resistivity and $\tilde{\chi}$ is given by

$$
\frac{\tilde{\chi}}{m+1}=\sup _{\alpha \in \mathbb{R}, \tau \in \mathbb{R}}\left\{\tau-\frac{\sqrt{3}}{2} \alpha-\frac{c_{1}}{3}\left(W_{1}^{*}-\alpha V\right)^{*}(\tau \boldsymbol{N})\right\} .
$$

The bound $\tilde{\chi}$ is non trivial for $m>1$, i.e. for $n<1$. Since $\chi_{\text {eff }}=\sigma_{\text {eff }}^{-1 / n}$, the lower bound $\tilde{\chi}$ on the effective resistivity translates as an upper bound on the effective conductivity $\sigma_{\text {eff. }}$ We have indeed

$$
\sigma_{\text {eff }} \leq \tilde{\chi}^{-n} .
$$

The upper bound given by (6.6) is represented in Fig. 10 along with the linear comparison bound $\sigma_{L C}$, given by (Ponte Castañeda, 1992)

$$
\frac{\sigma_{L C}}{\sigma_{1}}=\frac{c_{1}}{\left(2-c_{1}\right)^{\frac{(n+1)}{2}}} .
$$

For $n=0.1$, the relative improvement on the linear comparison bound $\sigma_{L C}$ is about $4 \%$.

\section{Concluding remarks}

Nonlinear Hashin-Shtrikman type bounds can be obtained by combining the translation method with the idea of embedding, whereby $n$ copies of the original problem are considered simultaneously in an extended problem of dimension $2 n$. In contrast with the linear case, the bounds obtained by taking $n=2$ are generally not optimal. In this paper, we investigate the choice $n=3$, thus considering an extended problem of dimension 6 .

Embedding the original problem in $\mathbb{R}^{6}$ leads to a genuine improvement of the bounds, but it comes at a price: The direct evaluation of the bounds is difficult to perform, even using numerical techniques. For isotropic constituents, invariance properties lead to considerable simplifications, thus making up for the increase in dimensionality that comes with the embedding approach. Ultimately those simplifications make for an efficient and reliable way of evaluating the bounds.

This paper concentrates on two-dimensional conductivity, but it should be mentioned that the results obtained apply to the mathematically equivalent problem of bounding the 


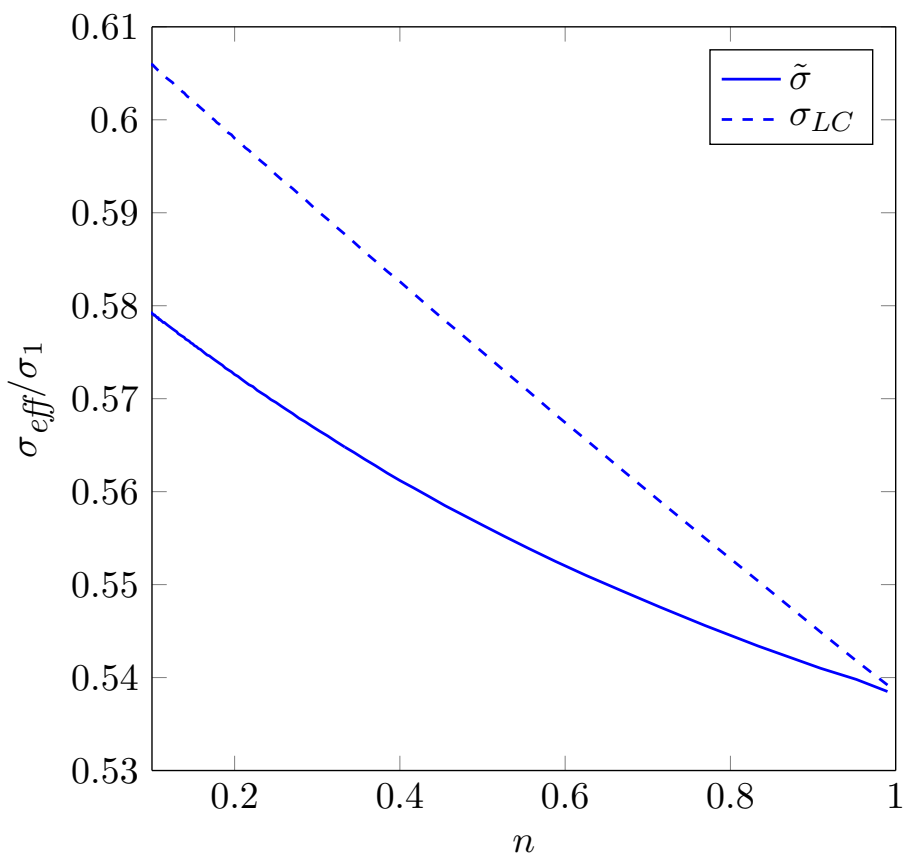

Figure 10: Upper bounds on the effective conductivity of a power-law composite, as a function of the nonlinearity index $n$. Case of infinite contrast, with $c_{1}=0.7$. Solid line: proposed bound. Dashed line: linear comparison bound.

elastic energy of fiber-reinforced composites under transverse shear. It is tempting to further extend the presented approach to three-dimensional conductivity or two-dimensional elasticity. For instance, in the case of three-dimensional conductivity, it can be expected that 4 copies of the original problem are necessary to improve on the linear comparison bound, thus leading to an extended problem of dimension 12. The issue of evaluating the corresponding bounds becomes even more critical, but the ideas used in this paper could be possibly be helpful to make the calculations tractable.

\section{Appendix A. Calculation of $g^{*}$ for dielectric breakdown}

In this Appendix we address the calculation of the function $g^{*}$ in (3.14). We have

$$
g^{*}(\tau)=\sup _{\boldsymbol{E} \in \mathcal{C}} g(\boldsymbol{E})
$$

where $\mathcal{C}=\left\{\boldsymbol{E}=\left(\boldsymbol{e}_{1}, \boldsymbol{e}_{2}, \boldsymbol{e}_{3}\right): e_{i} \leq 1\right\}$ and

$$
g(\boldsymbol{E})=\tau \boldsymbol{N} \cdot \boldsymbol{E}+\frac{1}{2} \boldsymbol{E} \cdot \boldsymbol{\mathcal { M }} \cdot \boldsymbol{E} .
$$


The problem A.1 is easily solved for $\tau=0$ : Since the largest eigenvalue of $\mathcal{M}$ is equal to $\sqrt{3}$, the supremum in A.1 is attained for any eigenvector of $\mathcal{M}$ (corresponding to the eigenvalue $\sqrt{3}$ ) of norm 3 and we have $g^{*}(0)=3 \sqrt{3} / 2$. We note that such an eigenvector if given by

$$
\frac{1}{2}(1, \sqrt{3},-1, \sqrt{3},-2,0)
$$

In the following, we address the general situation where $\tau \neq 0$. Let $\boldsymbol{E}$ attaining the supremum in A.1). We write $\boldsymbol{E}$ as $\boldsymbol{E}=\left(u_{1}, v_{1}, u_{2}, v_{2}, u_{3}, v_{3}\right)$ with $u_{i}^{2}+v_{i}^{2} \leq 1(i=1,2,3)$. The stationarity conditions give

$$
\begin{aligned}
\tau / 2+v_{2}+v_{3} & =\lambda_{1} u_{1} \\
-\sqrt{3} \tau / 2-u_{2}-u_{3} & =\lambda_{1} v_{1} \\
-\tau / 2-v_{1}+v_{3} & =\lambda_{2} u_{2} \\
-\sqrt{3} \tau / 2+u_{1}-u_{3} & =\lambda_{2} v_{2} \\
-\tau-v_{1}-v_{2} & =\lambda_{3} u_{3} \\
u_{1}+u_{2} & =\lambda_{3} v_{3}
\end{aligned}
$$

where the Lagrange multipliers $\lambda_{i}$ satisfy

$$
\lambda_{i} \geq 0, \lambda_{i}\left(u_{i}^{2}+v_{i}^{2}-1\right)=0
$$

The second-order optimality condition reads as

$$
0 \geq \frac{1}{2} \delta \boldsymbol{E} \cdot \boldsymbol{M} \cdot \delta \boldsymbol{E}+\sum_{i=1}^{3} \lambda_{i}\left(u_{i}, v_{i}\right) \cdot \delta \boldsymbol{e}_{i}
$$

for all $\delta \boldsymbol{E}=\left(\delta \boldsymbol{e}_{1}, \delta \boldsymbol{e}_{2}, \delta \boldsymbol{e}_{3}\right)$ verifying

$$
\left(u_{i}, v_{i}\right) \cdot \delta \boldsymbol{e}_{i} \leq 0 \text { if } u_{i}^{2}+v_{i}^{2}=1 .
$$

Using (A.3) and A.5), it can be proved that all the Lagrange multipliers $\lambda_{i}$ are non zero, i.e. that $u_{i}^{2}+v_{i}^{2}=1$ for $i=1,2,3$. The main argument is that if one of the Lagrange multipliers is equal to 0 then one can construct an eigenvector $\delta \boldsymbol{E}$ such that $\boldsymbol{M} \cdot \delta \boldsymbol{E}=\sqrt{3} \delta \boldsymbol{E}$ and $\lambda_{i}\left(u_{i}, v_{i}\right) \cdot \delta \boldsymbol{e}_{i}=0$ for $i=1,2,3$, which is in contradiction with A.5).

We now prove that, up to a rotation $\boldsymbol{\mathcal { R }}_{i}$ in $4.2, \boldsymbol{E}$ is of the form

$$
\boldsymbol{E}=(\cos \theta, \sin \theta,-\cos \theta, \sin \theta, \epsilon, 0)
$$

for some $\epsilon$ in $\{-1,1\}$. First assume that $\lambda_{1}+\lambda_{2}+\lambda_{3}-\lambda_{1} \lambda_{2} \lambda_{3} \neq 0$. In that case, the linear system A.3 can be inverted to give

$$
\left(\begin{array}{l}
u_{1} \\
v_{1} \\
u_{2} \\
v_{2} \\
u_{3} \\
v_{3}
\end{array}\right)=\frac{1}{2} \frac{\tau}{\lambda_{1}+\lambda_{2}+\lambda_{3}-\lambda_{1} \lambda_{2} \lambda_{3}}\left(\begin{array}{l}
\lambda_{3}\left(\sqrt{3}-\lambda_{2}\right) \\
\sqrt{3} \lambda_{2} \lambda_{3}-2 \lambda_{2}-\lambda_{3} \\
-\lambda_{3}\left(\sqrt{3}-\lambda_{1}\right) \\
\sqrt{3} \lambda_{1} \lambda_{3}-2 \lambda_{1}-\lambda_{3} \\
2 \lambda_{1} \lambda_{2}-\sqrt{3} \lambda_{1}-\sqrt{3} \lambda_{2} \\
\lambda_{1}-\lambda_{2}
\end{array}\right)
$$


Using the expressions A.7, the relation $u_{1}^{2}+v_{1}^{2}=u_{2}^{2}+v_{2}^{2}$ can be rewritten as

$$
0=\left(\lambda_{1}-\lambda_{2}\right) P\left(\lambda_{3}\right)
$$

where $P$ is the cubic polynomial defined by

$$
P(\lambda)=s-\sqrt{3}(s-\lambda) \lambda+(s-\lambda-\sqrt{3}) \lambda^{2}
$$

with $s=\lambda_{1}+\lambda_{2}+\lambda_{3}$. Similarly we have

$$
0=\left(\lambda_{1}-\lambda_{3}\right) P\left(\lambda_{2}\right)=\left(\lambda_{2}-\lambda_{3}\right) P\left(\lambda_{1}\right) .
$$

Calculating the discriminant of $P$ shows that $P$ only has one root in $\mathbb{R}$. Therefore, we deduce from (A.8) and A.10 that two of the multipliers $\lambda_{i}$ are necessarily equal. By applying one of the transformation $\boldsymbol{\mathcal { R }}_{i}$ in 4.2 , we can assume that $\lambda_{1}=\lambda_{2}$. The expressions A.7 rightly show that $v_{3}=0, u_{1}=-u_{2}, v_{1}=v_{2}$. Hence $\boldsymbol{E}$ is of the form A.6.

The above analysis needs to be modified if $\lambda_{1}+\lambda_{2}+\lambda_{3}-\lambda_{1} \lambda_{2} \lambda_{3}=0$. In that case, some manipulations on A.3 (that we do not report here) show that $\lambda_{1}, \lambda_{2}, \lambda_{3}$ are necessarily all equal to $\sqrt{3}$ and that $\boldsymbol{E}$ is parallel to $\boldsymbol{N}$ (and therefore of the form A.6).

The developments so far show that we only need to optimize the function in A.2 with respect to $\boldsymbol{E}$ of the form A.6. For such $\boldsymbol{E}$, a direct calculation gives

$$
g(\boldsymbol{E})=2(\cos \theta-\epsilon) \sin \theta+\tau(\cos \theta-\sqrt{3} \sin \theta-\epsilon) .
$$

Hence we are left with solving the scalar optimization problems $\sup _{\theta} g_{-}(\theta)$ and $\sup _{\theta} g_{+}(\theta)$ with

$$
\begin{aligned}
& g_{+}(\theta)=2(\cos \theta-1) \sin \theta+\tau(\cos \theta-\sqrt{3} \sin \theta-1), \\
& g_{-}(\theta)=2(\cos \theta+1) \sin \theta+\tau(\cos \theta-\sqrt{3} \sin \theta+1) .
\end{aligned}
$$

The stationarity condition $g_{ \pm}^{\prime}(\theta)=0$ gives

$$
\sin \theta=\frac{2}{\tau}\left(2 \cos ^{2} \theta-1-\epsilon \cos \theta\right)-\sqrt{3} \cos \theta
$$

with $\epsilon=1$ for $g_{+}$and $\epsilon=-1$ for $g_{-}$. Taking the square of A.11 leads to the polynomial equation

$$
0=16 x^{4}-8(2 \epsilon+\sqrt{3} \tau) x^{3}+4 x^{2}\left(\tau^{2}+\sqrt{3} \epsilon \tau-3\right)+4(2 \epsilon+\sqrt{3} \tau) x+4-\tau^{2}
$$

where $x=\cos \theta$. Observe from (A.11) that $x_{0}=-\epsilon / 2$ is a solution of (A.12). Factorizing the polynomial in A.12 by $(x+\epsilon / 2)$, we obtain that the solutions of A.12 (distinct from $-\epsilon / 2)$ are the roots of the cubic equation

$$
0=8 x^{3}+4(-3 \epsilon-\tau \sqrt{3}) x^{2}+\left(2 \tau^{2}+4 \sqrt{3} \epsilon \tau\right) x-\epsilon\left(\tau^{2}-4\right) .
$$


Setting $x=z+\epsilon / 2+\tau / 2 \sqrt{3}$, A.13 can be rewritten as

$$
0=8 z^{3}-6 z+\frac{\sqrt{3} \tau^{3}}{9}+2 \epsilon .
$$

It is necessary at this point to distinguish between the cases $\epsilon=1$ and $\epsilon=-1$.

For $\epsilon=-1$, the cubic equation (A.13) admits

$$
\begin{array}{ll}
\left.3 \text { solutions (denoted by } x_{3}>x_{2}>x_{1}\right) & \text { if } \tau<\tau_{1} \text {, } \\
\left.2 \text { solutions (denoted by } x_{2}>x_{1}\right) & \text { if } \tau=\tau_{1} \text {, } \\
1 \text { solution (denoted by } x_{1} \text { ) } & \text { if } \tau>\tau_{1} \text {; }
\end{array}
$$

where $\tau_{1}=\sqrt{3} 2^{2 / 3}$ (see Fig. A.11). Let $\theta_{i}$ be the value of $\theta$ satisfying $x_{i}=\cos \theta_{i}$ along with A.11. We need to find which of the values $g_{-}\left(\theta_{0}\right), g_{-}\left(\theta_{1}\right), g_{-}\left(\theta_{2}\right), g_{-}\left(\theta_{3}\right)$ is the largest. Setting $\tau_{0}=1+\sqrt{3}$, some manipulation shows that

$$
\sup g_{-}= \begin{cases}g_{-}\left(\theta_{3}\right) & \text { for } 0<\tau \leq \tau_{0} \\ g_{-}\left(\theta_{0}\right) & \text { for } \tau_{0} \leq \tau\end{cases}
$$

For $\epsilon=1$, A.13 only has one solution (denoted by $x_{1}^{\prime}$ ) in $\mathbb{R}$. Calculating the second derivative $g_{+}^{\prime \prime}$ shows that $x_{0}=-\epsilon / 2$ corresponds to a local minimum and that $x_{1}^{\prime}$ corresponds to a local maximum of $g_{+}$. It follows that

$$
\sup g_{+}=g_{+}\left(\theta_{1}^{\prime}\right)
$$

where $\theta_{1}^{\prime}$ satisfies $x_{1}^{\prime}=\cos \theta_{1}^{\prime}$ along with A.11). Comparing $g_{+}\left(\theta_{1}^{\prime}\right)$ with $\max \left(g_{-}\left(\theta_{0}\right), g_{-}\left(\theta_{3}\right)\right)$ shows that $\sup g_{+} \leq \sup g_{-}$. Hence $g^{*}(\tau)$ is equal to $\sup g_{-}$as given by (A.14.

\section{Appendix B. Additional details for a power-law behaviour}

For a power-law energy $w(e)=\frac{\sigma}{n+1} e^{n+1}$, the function $\mathcal{F}$ introduced in Eq. 4.24 specializes as

$$
\mathcal{F}(\boldsymbol{E})=\boldsymbol{T} \cdot \boldsymbol{E}+\frac{1}{2} \boldsymbol{E} \cdot \boldsymbol{\mathcal { M }} \cdot \boldsymbol{E}-\frac{\sigma}{n+1} \sum_{i=1}^{3} e_{i}^{n+1}
$$

where $\boldsymbol{T}=\tau \boldsymbol{N}, \boldsymbol{E}=\left(\boldsymbol{e}_{\mathbf{1}}, \boldsymbol{e}_{\mathbf{2}}, \boldsymbol{e}_{\mathbf{3}}\right)$ and $\boldsymbol{e}_{\boldsymbol{i}} \in \mathbb{R}^{2}$.

Note that $\mathcal{F}(0)=0$. In the following, we construct $R>0$ such that $\mathcal{F}(\boldsymbol{E}) \leq 0$ for any $\boldsymbol{E}$ verifying $\|\boldsymbol{E}\| \geq R$, so that

$$
\sup _{\boldsymbol{E}} \mathcal{F}(\boldsymbol{E})=\sup _{\boldsymbol{E}:\|\boldsymbol{E}\| \leq R} \mathcal{F}(\boldsymbol{E})
$$

As mentioned in Sect. 5.1, the property $(\mathrm{B} .2)$ is helpful for solving numerically the optimization problems that define the bounds. 


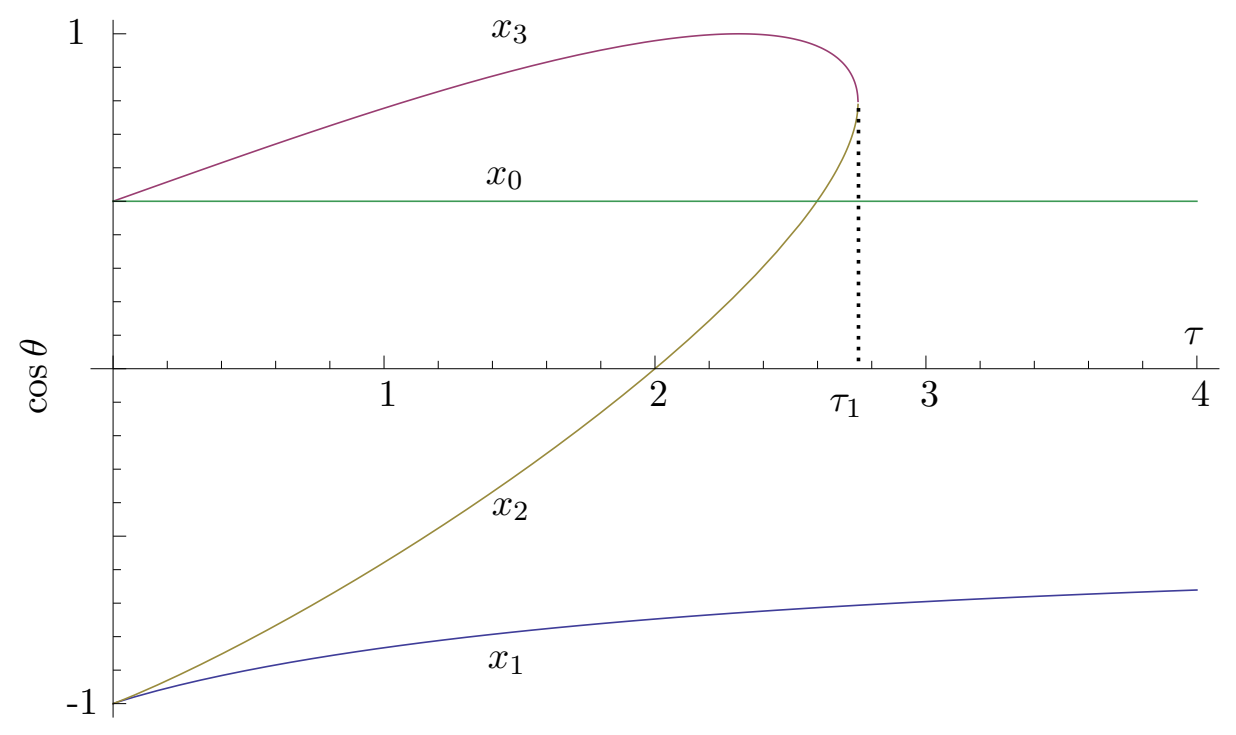

Figure A.11: Stationary points of $g_{-}$.

Since $\mathcal{M}$ is symmetric with eigenvalues 0 and $\pm \sqrt{3}$, we have $\boldsymbol{E} \cdot \mathcal{M} \cdot \boldsymbol{E} \leq \sqrt{3}\|\boldsymbol{E}\|^{2}$ for any $\boldsymbol{E}$. Therefore

$$
\mathcal{F}(\boldsymbol{E}) \leq\|\boldsymbol{T}\|\|\boldsymbol{E}\|+\frac{1}{2} \sqrt{3}\|\boldsymbol{E}\|^{2}-\frac{\sigma}{n+1} \sum_{i=1}^{3} e_{i}^{n+1} .
$$

where the Cauchy-Schwarz inequality has been used. We have the straightforward relations

$$
\sum_{i=1}^{3} e_{i}^{n+1} \geq \max \left(e_{1}^{n+1}, e_{2}^{n+1}, e_{3}^{n+1}\right)=\left(\max _{i} e_{i}\right)^{n+1}
$$

and

$$
\|\boldsymbol{E}\|^{2}=e_{1}^{2}+e_{2}^{2}+e_{3}^{2} \leq 3 \max \left(e_{1}^{2}, e_{2}^{2}, e_{3}^{2}\right)=3\left(\max _{i} e_{i}\right)^{2} .
$$

Combining the two preceding relations, we get

$$
\sum_{i=1}^{3} e_{i}^{n+1} \geq 3^{-\frac{n+1}{2}}\|\boldsymbol{E}\|^{n+1}
$$

Substituting in (B.3) yields

$$
\mathcal{F}(\boldsymbol{E}) \leq\|\boldsymbol{T}\|\|\boldsymbol{E}\|-\frac{1}{2} A(\|\boldsymbol{E}\|)\|\boldsymbol{E}\|^{2}
$$


where

$$
A(\|\boldsymbol{E}\|)=3^{-\frac{n+1}{2}} \frac{2 \sigma}{n+1}\|\boldsymbol{E}\|^{n-1}-\sqrt{3} .
$$

Since $\sigma>0$ and $n>1$, there exists $R_{0}>0$ such that $A(\|\boldsymbol{E}\|) \geq 1$ for any $\|\boldsymbol{E}\| \geq R_{0}$. Such a value of $R_{0}$ is given by

$$
R_{0}=3^{\frac{n+1}{2(n-1)}}\left[\frac{(n+1)(1+\sqrt{3})}{2 \sigma}\right]^{\frac{1}{n-1}} .
$$

For any $\boldsymbol{E}$ verifying $\|\boldsymbol{E}\| \geq R_{0}$, we obtain

$$
\mathcal{F}(\boldsymbol{E}) \leq\|\boldsymbol{T}\|\|\boldsymbol{E}\|-\frac{1}{2}\|\boldsymbol{E}\|^{2}=\frac{1}{2}\|\boldsymbol{E}\|(2\|\boldsymbol{T}\|-\|\boldsymbol{E}\|) .
$$

Hence $\mathcal{F}(\boldsymbol{E}) \leq 0$ for any $\boldsymbol{E}$ such that $\|\boldsymbol{E}\| \geq \max \left(R_{0}, 2\|\boldsymbol{T}\|\right)$. A value of $R$ satisfying (B.2) is thus given by

$$
R=\max \left(R_{0}, 2\|\boldsymbol{T}\|\right) .
$$

\section{References}

Bhattacharya, K., Kohn, R., 1997. Energy minimization and the recoverable strains in polycrystalline shape memory alloys. Arch. Ration. Mech. Anal. 139, 99-180.

Blumenfeld, R., Bergman, D. J., 1989. Exact calculation to second order of the effective dielectric constant of a strongly nonlinear inhomogeneous composite. Phys. Rev. B 40 (3), 1987-1989.

Blumenfeld, R., Bergman, D. J., 1991. Strongly nonlinear composite dielectrics: a perturbation method for finding the potential field and bulk effective properties. Phys. Rev. B 44 (14), 7378-7396.

Bruggeman, V. D., 1935. Berechnung verschiedener physikalischer konstanten von heterogenen substanzen. i. dielektrizitätskonstanten und leitfähigkeiten der mischkörper aus isotropen substanzen. Annalen der physik 416 (7), 636-664.

Fonseca, I., Müller, S., 1999. A-quasiconvexity, lower semicontinuity, and young measures. SIAM J. Math. Anal. 30 (6), 1355-1390.

Garroni, A., Kohn, R. V., 2003. Some three-dimensional problems related to dielectric breakdown and polycrystal plasticity. Proc. R. Soc. London A 459 (2038), 2613-2625.

Hashin, Z., Shtrikman, S., 1962. A variational approach to the theory of the effective magnetic permeability of multiphase materials. J.Appl.Phys. 33 (10), 3125-3131. 
Hill, R., 1963. Elastic properties of reinforced solids: some theoretical principles. J. Mech. Phys. Solids 11 (5), 357-372.

Idiart, M. I., Ponte Castañeda, P., 2013. Estimates for two-phase nonlinear conductors via iterated homogenization. Proc. R. Soc. London A 469 (2153), 20120626.

Kohn, R. V., Little, T. D., 1998. Some model problems of polycrystal plasticity with deficient basic crystals. SIAM J. Appl. Math. 59 (1), 172-197.

Lurie, K., Cherkaev, A., 1984. Exact estimates of conductivity of composites formed by two isotropically conducting media taken in prescribed proportion. Proc. R. Soc. Edinburgh A $99(1-2), 71-87$.

Maxwell-Garnett, J. C., 1904. Colours in metal glasses and in metal films. Philos. Trans. R. Soc. London 203, 385-420.

Milton, G. W., 1990. On characterizing the set of possible effective tensors of composites: the variational method and the translation method. Commun. Pure Appl. Math. 43 (1), $63-125$.

Milton, G. W., 2002. The Theory of Composites. Cambridge University Press.

Milton, G. W., Serkov, S. K., 2000. Bounding the current in nonlinear conducting composites. J. Mech. Phys. Solids 48 (6-7), 1295-1324.

Murat, F., Tartar, L., 1985. Calcul des variations et homogénéisation. Les méthodes de l’homogénéisation: théorie et applications en physique 57, 319-369.

Nesi, V., Smyshlyaev, V., Willis, J. R., 2000. Improved bounds for the yield stress of a model polycrystalline material. J. Mech. Phys. Solids 48 (9), 1799-1825.

Nesi, V., Talbot, D. R. S., Willis, J. R., 1999. Translation and related bounds for the response of a nonlinear composite conductor. Proc. R. Soc. London A 455 (1990), 36873707 .

Norris, A., Callegari, A., Sheng, P., 1985. A generalized differential effective medium theory. J. Mech. Phys. Solids 33 (6), 525-543.

Peigney, M., 2005. A pattern-based method for bounding the effective response of a nonlinear composite. J. Mech. Phys. Solids 53 (4), 923-948.

Peigney, M., 2008. Recoverable strains in composite shape-memory alloys. J. Mech. Phys. Solids 56, 360-375.

Peigney, M., 2016. Improved bounds on the energy-minimizing strains in martensitic polycrystals. Continuum Mech. Thermodyn. 28 (4), 923-946. 
Ponte Castañeda, P., 1991. The effective mechanical properties of nonlinear isotropic solids. J. Mech. Phys. Solids 39, 45-71.

Ponte Castañeda, P., 1992. Bounds and estimates for the properties of nonlinear heterogeneous systems. Phil. Trans. R. Soc. London A 340 (1659), 531-567.

Ponte Castañeda, P., deBotton, G., Li, G., 1992. Effective properties of nonlinear inhomogeneous dielectrics. Phys. Rev. B 46, 4387-4394.

Ponte Castañeda, P., 2002. Second-order homogenization estimates for nonlinear composites incorporating field fluctuations: I-theory. J. Mech. Phys. Solids 50 (4), 737-757.

Ponte Castañeda, P., 2012. Bounds for nonlinear composites via iterated homogenization. J. Mech. Phys. Solids 60, 1583-1604.

Ponte Castañeda, P., Kailasam, M., 1997. Nonlinear electrical conductivity in heterogeneous media. Proc. R. Soc. London A 453 (1959), 793-816.

Ponte Castañeda, P., Willis, J. R., 1993. The effective behavior of nonlinear composites: a comparison between two methods. In: Materials Science Forum. Vol. 123. Trans Tech Publ, pp. 351-360.

Rockafellar, R. T., 1970. Convex Analysis. Princeton Mathematical Series.

Talbot, D. R. S., Willis, J. R., 1985. Variational principles for inhomogeneous non-linear media. IMA J. Appl. Math. 35 (1), 39-54.

Talbot, D. R. S., Willis, J. R., 2004. Bounds for the effective constitutive relation of a nonlinear composite. Proc. R. Soc. London A 460 (2049), 2705-2723.

Tartar, L., 1985. Estimations fines des coefficients homogénéisés. In: Krée, P. (Ed.), Ennio de Giorgi Colloquium: Papers presented at the H.Poincaré Institute in November 1983. Pitman Publishing, pp. 168-187.

Wiener, O. H., 1912. Die theorie des mischkörpers für das feld der stationären strömung. 1. abhandlung: Die mittelwertsätze für kraft, polarisation und energie. BG Teubner.

Willis, J. R., 1977. Bounds and self-consistent estimates for the overall properties of anisotropic composites. J. Mech. Phys. Solids 25 (3), 185-202.

Willis, J. R., 1986. Variational estimates for the overall response of an inhomogeneous nonlinear dielectric. In: Homogenization and effective moduli of materials and media. Springer, pp. 247-263. 